\title{
Mixing Nonlocality and Mixing Anisotropy in an Idealized Western Boundary Current Jet
}

\author{
RU CHEN ${ }^{\mathrm{a}}$ \\ Scripps Institution of Oceanography, University of California, San Diego, La Jolla, California
}

STEPHANIE WATERMAN

Department of Earth, Ocean and Atmospheric Sciences, University of British Columbia, Vancouver, British Columbia, Canada

(Manuscript received 27 January 2017, in final form 21 October 2017)

\begin{abstract}
Motivated by the key role of western boundary currents in shaping water mass distribution and gyre water exchanges, this study characterizes mixing in an idealized western boundary current jet using a barotropic quasigeostrophic model with numerical particles deployed. Both the nonlocality of mixing, depicted by nonlocality ellipses, and mixing anisotropy, depicted by mixing ellipses, are estimated. Mixing is more nonlocal within the jet compared to the jet flanks. In general, the size of nonlocality ellipses, a metric of the degree of mixing nonlocality, scales with the eddy velocity magnitude and the equilibration time for diffusivity. The tilt and eccentricity of the nonlocality ellipses, a characterization of the anisotropy of mixing nonlocality, agree with those of momentum flux ellipses in the regions where mixing nonlocality is small. Mixing ellipse characteristics are flow regime dependent. In regions dominated by wave radiation, the mixing ellipses align with the contours of the wave streamfunction and are very anisotropic. Inside the recirculations, however, the mixing ellipses are nearly isotropic. Mixing ellipses are zonally elongated in the jet upstream because of the suppression of cross-jet mixing by the jet and the anisotropy of eddy velocity, and they can have negative minor axis length in the jet downstream, indicating negative cross-jet eddy diffusivity, which is consistent with upgradient eddy fluxes there. Thus, despite significant spatial heterogeneity in mixing nonlocality and anisotropy, in this idealized system at least, spatial patterns in these diagnostics tend to be relatively large scale and tied to larger-scale dynamics. The implications of these results to eddy parameterization and jet dynamics are discussed.
\end{abstract}

\section{Introduction}

Oceanic jets are prevalent in the World Ocean and play an important role in the climate system. Jets in some oceanic regions are latent, that is, only manifest themselves in the time-mean flow field, such as those in the central Pacific (e.g., Maximenko et al. 2005; Kamenkovich et al. 2009; Berloff et al. 2009; Chen et al. 2015a). Others are intense and clearly visible in the flow snapshots, with notable examples in the energetic oceanic regions such as in the western boundary current (WBC) extensions and in the Antarctic Circumpolar Current (ACC). These intense

\footnotetext{
${ }^{\text {a }}$ Current affiliation: Department of Atmospheric and Oceanic Sciences, University of California, Los Angeles, Los Angeles, California.
}

Corresponding author: Ru Chen, ruchen@alum.mit.edu jets have extremely large along-jet transports, are an importance energy source to the eddy field through instability processes, and can serve as cross-jet transport barriers (Bower et al. 1985; Gille et al. 2007; Thompson 2008; Chen et al. 2014a,b, 2016). As a consequence, these jet flows greatly regulate the global tracer and energy budgets and thus climate variability.

Unsurprisingly, many studies exist about the dynamics and consequences of these strong jets. Topics include, but are not limited to, their spatiotemporal structures, interaction with topography, and energy exchange with eddies (e.g., Watts et al. 1995; Killworth and Hughes 2002; Howe et al. 2009; Jayne et al. 2009; Thompson 2010; Qiu and Chen 2010; Venaille et al. 2011; Waterman et al. 2011; Thompson and Sallée 2012; Tracey et al. 2012; Chen et al. 2014a). In particular, transport and mixing across jets, as well as the role of jets in regulating cross-jet transport and mixing, have received much attention 
recently, as cross-jet mixing is critical to the meridional transport of heat and other tracers across the ACC and WBCs. For example, Samelson (1992) evaluated the effect of the frequency of meander fluctuations in cross-jet exchange, and Rypina et al. (2011) characterized the important role of the Gulf Stream in the fluid exchange between subtropical and subpolar gyres. Further, the horizontal and vertical structures of isopycnal eddy diffusivities across the jets have been estimated in the Southern Ocean, Kuroshio Extension (KE) regions, and idealized circumpolar current by multiple studies (e.g., Gille et al. 2007; Abernathey et al. 2010; Griesel et al. 2014; Chen et al. 2014b; LaCasce et al. 2014; Wolfram and Ringler 2017). Much theoretical effort, based on the critical layer concept from Green (1970), has also been made toward quantifying the role of the jet in cross-jet mixing (e.g., Ferrari and Nikurashin 2010; Klocker et al. 2012a; Bates et al. 2014; Chen et al. 2015b).

The full diffusivity tensor $\kappa_{i j}$, in addition to the diffusivity in the cross-jet direction, is important for characterizing eddy mixing rates. In the Eulerian framework, the Eulerian eddy diffusivity tensor $\kappa_{i j}^{E}$ is defined as a mixing coefficient that relates the eddy tracer fluxes to the mean tracer gradient in the form

$$
u_{i}^{\prime} C^{\prime}=-\kappa_{i j}^{E} \frac{\partial}{\partial x_{j}} \bar{C}
$$

where $u_{i}^{\prime} C^{\prime}$ are the eddy tracer fluxes with $u_{i}^{\prime}$ and $C^{\prime}$ as the eddy velocity and eddy tracer perturbations, respectively, and $\bar{C}$ denotes the mean tracer concentration. The meridional overturning circulation and climate variability in coarse-resolution models are sensitive to the specification of this mixing coefficient (e.g., Danabasoglu and Marshall 2007). Since the full diffusivity tensor, and not just its cross-jet component, needs to be implemented in coarseresolution climate models, characterizing and understanding $\kappa_{i j}$ is no less important than characterizing and understanding the diffusivity's cross-jet component for the task of representing eddy mixing effects in models.

Mixing ellipses, derived from the symmetric part of $\kappa_{i j}$, are a useful tool to reveal the dominant magnitude, anisotropy, and direction of eddy mixing rates. Using altimeter observations of sea surface height, Rypina et al. (2012) estimated mixing anisotropy in the North Atlantic. Mixing ellipses have also been depicted in an idealized circumpolar channel (Abernathey et al. 2013) and midlatitude basin models (Wolfram et al. 2015). However, studies characterizing $\kappa_{i j}$ and mixing ellipses in the WBC jet regions are lacking. In particular, the factors controlling mixing anisotropy, anticipated to be large in zonally elongated, highly anisotropic systems like WBC jets, remain largely unclear. Furthermore, theories built upon the critical layer concept are mostly used to discuss the role of the jets in regulating cross-jet mixing, not the full diffusivity tensor (e.g., Green 1970; Ferrari and Nikurashin 2010; Klocker et al. 2012a; Bates et al. 2014; Chen et al. 2015b). Motivated by these gaps, this study aims to estimate the full diffusivity tensor $\kappa_{i j}$ and its corresponding mixing ellipses in an idealized model of a WBC jet and in particular to assess the role of the jet in shaping the anisotropy of the mixing. As summarized in the abstract, we find that mixing ellipse characteristics differ much in the three dynamical regimes of an idealized barotropic jet system (jet area, recirculation gyre, and wave radiation regime). Details and corresponding dynamics are provided in section 5 .

In addition to estimating the anisotropy of mixing via the diagnosis of the full diffusivity tensor and its corresponding mixing ellipses, we also aim to quantify the nonlocality of mixing in WBC jet regions. One assumption inherent in the diffusivity/parameterization concept is that eddy fluxes can be represented by a diffusivity coefficient multiplied by a local tracer gradient. However, expanding eddy tracer fluxes into a form employing Green functions clearly reveals that the eddy tracer flux at a given point depends on both local and nonlocal large-scale tracer gradients (e.g., Kraichnan 1987; Chen et al. 2015b). Further, Lagrangian eddy diffusivities are also inherently nonlocal because diagnosing Lagrangian diffusivities involve the integration of a Lagrangian autocorrelation function over a finite time period, and thus diffusivities are derived from particle trajectories that span a finite area (Rypina et al. 2012; Chen et al. 2014b). It is important to access the nonlocality of mixing to evaluate the validity of the local assumption inherent in Eq. (1), to intelligently interpret our diagnoses of the Lagrangian diffusivity in heterogeneous systems, and to consider the practicalities of including nonlocality in future eddy parameterizations. As a first step toward these goals, in this study we depict and discuss the factors controlling the characteristics of mixing nonlocality in an idealized WBC jet. In this configuration, we hypothesize that mixing inside the jet is significantly nonlocal because of the strong advection by the intense jet flow.

To evaluate the anisotropy of the diffusivity tensor and its nonlocality in WBC jets, we chose to use a highly simplified, idealized model, specifically a barotropic, quasigeostrophic $(\mathrm{QG})$ model of a free inertial jet. In reality, WBCs have complex spatiotemporal characteristics influenced by many factors (e.g., coastline curvature, topography, remote wind forcing, and low-frequency oscillations between stable and unstable states; see, e.g., Qiu and Chen 2010; da Silveira 2013; Chen et al. 2014b). We are motivated to use a simple model that does not include these complicating influences instead of a realistic eddying 
general circulation model that does to allow us to focus on basic mixing dynamics inherent to these jet systems generally. A series of previous studies (e.g., Waterman and Jayne 2011; Waterman and Hoskins 2013) have demonstrated that this model, though idealized, is a useful tool and can lead to dynamical insights relevant to realistic WBCs. For example, the model has been used to elucidate the eddy-mean flow interaction processes that allow eddies to extract energy from the mean flow in the upstream region of the jet and feed energy back to the mean flow in the downstream region of the jet. This phenomenon is also found in both a realistic global eddying state estimate and satellite observations (e.g., Chen et al. 2014a).

The diffusivity tensor $\kappa_{i j}$ can be estimated from a number of different methods including the multitracer method (e.g., Bachman and Fox-Kemper 2013), the particle approach (e.g., Chen et al. 2014b), and the special tracer approach (Chen and Flierl 2015), among others. In this study, we deploy numerical particles in our idealized model and infer $\kappa_{i j}$ from the particle trajectories. Compared to the methodology of Chen et al. (2014b), our mixing diagnosis approach is novel in that we infer more local mixing rates and we remap the diffusivities to take into account mixing nonlocality (see section 3 ). The more local mixing rates are obtained because of using equilibration time in the calculation of $\kappa_{i j}$ (section 3 ).

This paper is organized as follows: Section 2 introduces the idealized WBC jet model and describes the method employed to obtain numerical particle trajectories in the modeled jet flow. Section 3 presents the methods used to estimate the nonlocality and anisotropy of mixing from these trajectories. Section 4 estimates and interprets the nonlocality of mixing in this system by characterizing and interpreting the properties of the nonlocality ellipses. A simple scaling predicting the size of the nonlocality ellipse is provided. The nonlocality ellipses are also compared with momentum flux ellipses, a related, but inherently Eulerian diagnostic. In section 5, we characterize and interpret the mixing ellipses diagnosed in the model as a function of the various dynamical flow regimes. Section 6 provides a summary and discussion of our results and conclusions.

\section{Model description}

\section{a. Idealized WBC jet model}

The idealized WBC jet model we employ is a nondimensionalized, barotropic, QG model of a freely evolving, unstable, inertial jet forced by a specified jet profile imposed at the western boundary of the study domain. This model has been extensively studied by Waterman and Jayne (2011), Waterman and Hoskins (2013), and Waterman and Lilly (2015), in which the model setup and its relevance to a typical real-world
WBC jet have been described in detail. In brief, the model is governed by the equation for the conservation of potential vorticity in barotropic flows:

$$
\frac{\partial}{\partial t} q+J(\psi, q)=-R \nabla^{2} \psi
$$

where $q$ is the barotropic potential vorticity (PV) $q=\nabla^{2} \psi+\beta y$. Here, $\psi, \beta$, and $R$ are the nondimensional values of the streamfunction, the meridional gradient of planetary vorticity, and bottom friction coefficient, respectively. The quantity $\beta$ is equal to $\beta_{\operatorname{dim}} L^{2} / U$, where $\beta_{\mathrm{dim}}$ is the dimensional meridional gradient of the Coriolis parameter, and $L$ and $U$ are dimensional scalings of the model, commonly taken to scale the width and velocity magnitude of the inflowing jet. A choice of $L=40 \mathrm{~km}$ and $U=1 \mathrm{~m} \mathrm{~s}^{-1}$ makes the model jet's dimensionalized jet scales typical of WBC jet values. In the specific model run considered here, the nondimensional $\beta$ parameter is set to 0.05 , and $R$ is $1 \times 10^{-6}$. For reference, the Gulf Stream has a value of nondimensional $\beta$ in the range of $0.02-0.13$ (Flierl et al. 1987), and a nondimensional value of $R$ of this magnitude implies a dissipation time scale of the order of 10 years. The width and length of the numerical domain are 150 and 80 nondimensional length units (ndlus), respectively, with highly dissipative sponge layers of width 10-20 ndlus set at all four lateral boundaries. This domain size allows for the upstream dynamics, which we study (the full development of eddies generated by the jet's instability and the subsequent stabilization of the jet by these eddies and a regime of wave radiation downstream) to be insensitive to both the domain size and the lateral and downstream boundary conditions. The sponges allow us to simulate open-ocean conditions, dissipating the energy radiated from the jet as opposed to allowing it to reflect back into the interior. Results presented in this work are based on model output for 5000 nondimensional time units (ndtus) at a temporal interval of 1 ndtu in the equilibrated state. They thus are representative of the dynamics of the statistically steady state of the turbulent flow.

Figure 1 shows the time-mean circulation and eddy kinetic energy (EKE) patterns in the model run we analyze in this study, which are similar to Fig. 3a in Waterman and Lilly (2015). Here, the time-evolving flow is turbulent and its energy source is the barotropically unstable jet imposed at the western boundary. The time-mean circulation is characterized by two recirculation gyres respectively located at the northern and southern flank of the strong zonal jet in the upstream region of the domain. Both the instantaneous and time-mean jet flow is strong in the upstream region $(x \leq 30)$. Downstream of this location, it gradually gets weaker as $x$ increases. The strongest eddy kinetic energy 


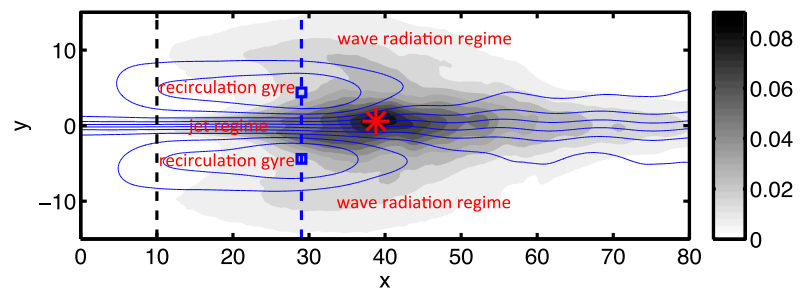

FIG. 1. Key features of the idealized WBC jet model, with the blue contours denoting the time-mean streamfunction. Three regimes (red labels) exist here: the jet, the recirculation gyre, and the wave radiation regime. The blue dashed line, which links the two maximum absolute values of the time-mean streamfunction (blue squares), indicates the zonal location of the maximum time-mean jet transport, which is a useful proxy for the downstream location of the time-mean jet's unstable-stable boundary (Waterman and Jayne 2011). Gray shading shows the temporal average of EKE $\left[\overline{0.5\left(u^{\prime 2}+v^{\prime 2}\right)}\right]$, with the red star pointing to its maximum value. The temporal average here denotes the average over 5000 ndtus in the equilibrated state. The western sponge layer is located at $0<x<10$. The dashed black line indicates the boundary of the western sponge layer

occurs on the jet axis between $x \sim 30$ and $x \sim 50$, where the jet is relatively weak.

The dominant eddy-mean flow interaction processes in this model have been extensively examined in previous studies (e.g., Waterman and Jayne 2011; Waterman and Hoskins 2013; Waterman and Lilly 2015) and include eddy generation by the jet's instability, the stabilization of the jet by these eddies after they have grown to sufficient amplitude, and the eddy strengthening of the time-mean jet in a downstream region of localized wave radiation from the meandering jet. These previous works reveal that from the perspective of eddy properties and dominant mechanisms of eddy-mean flow interaction, it is useful to characterize this system as being composed of three distinct dynamical regimes: the jet, the two recirculation gyres on the jet flanks, and the wave radiation regime in the remaining part of the domain (see Fig. 1).

\section{b. Particle trajectory calculations}

Both tracer-based and particle-based approaches to characterizing the eddy diffusivity have been employed in previous mixing studies (see, e.g., Abernathey et al. 2013). The tracer-based approach introduced by Nakamura (1996) can usefully infer effective diffusivities across the tracer contours by calculating the evolution of tracer contours with time. However, its estimation of mixing rate across tracer contours has very low spatial resolution, and it cannot be used to estimate mixing rates along tracer contours or the full diffusivity tensor $\kappa_{i j}$ (e.g., Shuckburgh et al. 2009; Abernathey et al. 2010). To overcome these limitations, one can use multiple tracers with different initial tracer gradients, allowing one to estimate all the components of $\kappa_{i j}$ based on the flux-gradient relation.
However, diffusivities from this approach are sensitive to the specification of the initial tracer concentrations (e.g., Bachman 2012; Bachman and Fox-Kemper 2013; Plumb and Mahlman 1987). Many studies have demonstrated that the single-particle approach to characterizing eddy diffusivity is useful for obtaining converged diffusivity estimates in any direction in both realistic and idealized scenarios (e.g., Oh et al. 2000; Zhurbas and Oh 2003; Katsumata and Yoshinari 2010; Qian et al. 2013; Chiswell 2013; Chen et al. 2014b, 2015b; Griesel et al. 2010), assuming a large number of particle trajectories is considered (e.g., Klocker et al. 2012b; Chen et al. 2014b). We chose to use this approach in this study.

Following Sallée et al. (2011), we use an offline particle advection approach to generate particle trajectories in the model flow. The particles are released at a spatial resolution of $0.2 \mathrm{ndlu} \times 0.2 \mathrm{ndlu}$ in the entire model domain and then advected by the total Eulerian velocities using the Runge-Kutta time-stepping scheme. Since particles in this work are advected by the total flow, the effect of mean flow on mixing is taken into account. We use a time step for the particle advection of $0.01 \mathrm{ndtu}$, which is a compromise between accuracy and computing constraints. Particle trajectories change little if we further decrease this time interval. Since the velocity fields are saved at model grid points at a temporal resolution of 1 ndtu, the velocities at the particle position at each time step are obtained through linear interpolation of the saved velocities in the spatial-temporal domain. In each ensemble run of the particle advection, these particles are advected for $500 \mathrm{ndtu}$, and this procedure is repeated 22 times at every 200 ndtus using the 5000-ndtu saved Eulerian model output. This yields a total of 22 ensembles of 500-ndtu trajectories of particles released throughout the model domain at a spatial resolution of 0.2 ndlu $\times 0.2$ ndlu.

Figure 2 shows the total number of days that particles spend in each $2 \times 1$ ndlu bin. The particle day counts are $O\left(10^{5}\right)$, which are large enough for us to obtain converged diffusivity estimates. Although particles are deployed uniformly in the entire domain, the spatial variability of the total number of particle days is large. For example, the number of particle days is small in the upstream region of the jet because the particles are advected out of the bin quickly by the intense jet flow. In contrast, this count is relatively large in the recirculation gyre regions where the mean circulation is weak.

\section{Methodology for mixing estimation}

\section{a. Estimating the eddy diffusivity tensor}

\section{1) Diagnostic Formula}

The single-particle diagnostic approach, developed by Davis $(1987,1991)$, has been successfully applied in a 


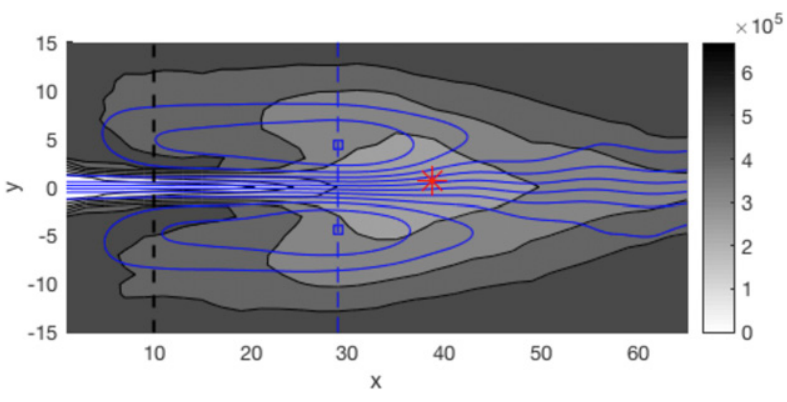

FIG. 2. The total number of days (gray shading) that particles in the 22 ensembles spend in each geographic bin of size 2 ndlus in the $x$ direction and 1 ndlu in the $y$ direction. Annotations denoting the location of the maximum EKE, the maximum absolute values of the time-mean streamfunction, the zonal location of maximum time-mean jet transport/the time-mean jet's unstable-stable boundary, and the extent of the western sponge layer are as in Fig. 1.

large number of mixing studies (e.g., Oh et al. 2000; Zhurbas and Oh 2004; Katsumata and Yoshinari 2010; Griesel et al. 2010; Qian et al. 2013; Chiswell 2013; Chen et al. 2014b, 2017). Following this approach, we diagnose the eddy diffusivity tensor at location $\mathbf{x}, \kappa_{i j}^{\infty}(\mathbf{x})$, using the following formula:

$$
\kappa_{i j}^{\infty}(\mathbf{x})=\lim _{\tau \rightarrow \infty} \kappa_{i j}(\mathbf{x}, \tau) \approx \frac{\int_{\tau_{1}}^{\tau_{2}} \kappa_{i j}(\mathbf{x}, \tilde{\tau}) d \tilde{\tau}}{\tau_{2}-\tau_{1}},
$$

where

$$
\kappa_{i j}(\mathbf{x}, \tau)=\int_{0}^{\tau} d \tilde{\tau}\left\langle u_{i}^{\prime}\left(t_{0} \mid \mathbf{x}, t_{0}\right) u_{j}^{\prime}\left(t_{0}+\tilde{\tau} \mid \mathbf{x}, t_{0}\right)\right\rangle_{L} .
$$

As described by Chen et al. (2014b), $u_{j}^{\prime}\left(t_{0}+\tau \mid \mathbf{x}, t_{0}\right)$ is the eddy velocity at time $t_{0}+\tau$ at the location of the particle, which passes location $\mathbf{x}$ at time $t_{0}$. Eddy velocity in this study denotes the deviation of the total velocity from its time mean over the entire available 5000-ndtu record. The term $\langle\cdot\rangle_{L}$ denotes averaging over all the particles passing through a specific area, that is, the bin, centered at $\mathbf{x}$. Our choice of $\left[\tau_{1}, \tau_{2}\right]$, which is different from that in Chen et al. (2014b), is discussed in section 3a(4).

More details about Eqs. (3) and (4), which have been employed in a series of previous studies about mixing in the regions with jets, are available in Griesel et al. (2010, 2014). The fundamental idea of inferring diffusivity from particle dispersion dates back to Taylor (1922). Taylor (1922) focuses on the dispersion in isotropic and homogeneous turbulence and this topic is extensively documented in chapter 5 of Monin and Yaglom (1971). Our diagnostic framework is based on Davis (1987, 1991), who essentially extends the idea of Taylor (1922) to the case with inhomogeneity and mean flow. Note that $u_{j}^{\prime}\left(t_{0}+\tau \mid \mathbf{x}, t_{0}\right)$ from Eq. (4) essentially denotes velocities of the particles subtracted from the "local" timemean Eulerian flow, not a spatially uniform mean flow. This choice can minimize the dispersion from mean flow shear and lead to improved convergence properties (Griesel et al. 2010, 2014; Chen et al. 2014b). Readers who are interested in shear dispersion can refer to Young et al. (1982) and Rhines and Young (1983), who analytically revealed interesting features of shear flow dispersion in the context of closed streamlines, internal waves, and so on.

\section{2) Pseudoparticle trajectories}

Following a series of previous studies (e.g., Davis 1991; Griesel et al. 2010; Klocker et al. 2012b; Chen et al. 2014b), we chose to analyze pseudoparticle trajectories. Specifically, we consider particle positions at every 20 ndtus as a starting point of a new trajectory and then track the particle forward for 150 ndtus. Illuminating diagrams to illustrate the concept of pseudotrajectories are available in Griesel et al. (2010) and Klocker et al. (2012b) and thus not included here. Our calculation of $\langle\cdot\rangle_{L}$ in Eq. (4) is essentially the average over these pseudotrajectories.

The approach of pseudotrajectories has the following advantages (Chen et al. 2014b). First, this method greatly increases the number of trajectories available for the diffusivity estimates and thus one can obtain converged diffusivity estimates at higher spatial resolution. Second, note that eddy diffusivities estimated from trajectories starting at $t_{0}$ essentially represent eddy diffusivities for the time period $t_{0} \in\left[t_{0}, t_{0}+\tau_{2}\right]$ [Eq. (3)]. Pseudotrajectories consider the particle positions every 20 ndtus as a new starting point $t_{0}$, whereas the original trajectories only consider particle positions every 200 ndtus as a new starting point $t_{0}$ (section 2b). Since each particle trajectory contributes to multiple pseudotrajectories, diffusivities inferred from pseudotrajectories are essentially the average over a much larger number of members at a much finer temporal resolution (i.e., every 20 ndtus). Therefore, the average of eddy diffusivities from the pseudotrajectories, compared to that from original trajectories, more accurately represent the time-mean mixing structure.

In our diffusivity estimation, pseudotrajectories containing points in the sponge layers are not used, as the high damping rates in the sponges are not necessarily relevant to real-world western boundary current dynamics, and thus including these tracks could lead to biases in our mixing estimation. In contrast to Chen et al. (2014b), we use only forward, not backward, pseudotracks because in the upstream region of the jet where advection by the jet is large, the particles are advected downstream so fast that 
there are no backward tracks with a length of 150 ndtus and a starting point in the upstream jet region. Though we present results based on pseudotrajectories by selecting a new starting point every 20 ndtus, we have also reduced the number to every 10 ndtus and every 5 ndtus and found that our estimates are not sensitive to this change.

\section{3) Choice of AVeraging bins: Adaptive VERSUS GEOGRAPHIC}

To obtain converged diffusivity estimates, we need to average the Lagrangian autocorrelation in Eq. (4) over a region with finite area (i.e., a bin) that contains an adequate number of pseudotrajectories. One issue is how to divide the domain into bins in such a way as to ensure adequate convergence of the diffusivity estimates while maintaining as high a spatial resolution in the diffusivity estimate as possible. One natural choice is to divide the domain into regular geographic bins (e.g., McClean et al. 2002; Zhurbas and Oh 2003). That is, one divides the entire study domain into rectangular boxes, with the same size and shape. Note that although particles are uniformly deployed in the study domain, the number of particle days in each regular geographic bin has large spatial variability (e.g., small in the intense jet) because of the flow inhomogeneity (not shown). Thus, the number of pseudotrajectories in each geographic bin can differ greatly. In this case, the uncertainties of diffusivity estimates have large variability among different bins, and the percentage of bins with converged diffusivity estimates can be low. This issue has been discussed in detail in Chen et al. (2014b). To address it, following Chen et al. (2014b), we chose to use the so-called adaptive bins originating from Koszalka and LaCasce (2010).

Compared to geographic bins, the shape of adaptive bins is irregular and their size can have large spatial variability. However, by construction, the number of pseudotrajectories in each adaptive bin is roughly uniform. Compared to using geographic bins, the use of adaptive bins yields diffusivity estimates with more consistent convergence and also more uniform uncertainty (e.g., Koszalka and LaCasce 2010; Chen et al. 2014b). Figure 3 shows the distribution of the adaptive bins we compute for our study domain. The adaptive bins are constructed using the starting points of these pseudotrajectories following the clustering algorithm from Koszalka and LaCasce (2010). For 71\% of the adaptive bins, the number of pseudotrajectories contained ranges from 1300 to 1700 , and for $94 \%$ of the adaptive bins, it ranges from 1200 to 1800 . This visualization reveals that there is significant spatial heterogeneity in the adaptive bin distribution in this system. Adaptive bins are relatively sparse in the upstream jet region, as the number of particles within the jet is

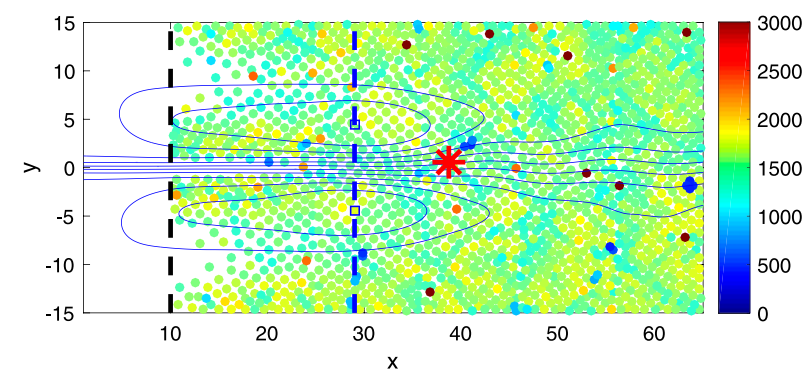

FIG. 3. The location of the adaptive bins generated for the diffusivity estimate (colored dots). The color of each dot indicates the number of pseudotracks in each bin. Annotations denoting key features of the time-mean circulation and EKE distribution are as in Fig. 1.

smaller than that outside of the jet because of the fast advection of particles by the jet flow (Fig. 2). The number of bins located near $x=10$ and $|y|=10$ is also small, as in these areas; some pseudotracks enter the sponge layer because of the westward advection of the recirculation gyres and are thus excluded in the bin generation and mixing estimation.

\section{4) INTEGRATION TIME}

Another issue is how to choose the time integration limits in the diffusivity calculation, that is, $\left[\tau_{1}, \tau_{2}\right]$ in Eq. (3). While Chen et al. (2014b) arbitrarily chose $\left[\tau_{1}, \tau_{2}\right]$ to be $[50,69]$ days, here we chose it to be a function of the equilibration time of $\kappa_{i j}$ and $\tau_{\text {eq }}$, specifically [ $\tau_{\text {eq }}-15$ ndtus, $\tau_{\mathrm{eq}}+15$ ndtus]. In this way, the integration limits of the diffusivity tensor changes with both the location and the component of the diffusivity tensor in question. Although diffusivity is a nonlocal concept, as it involves integrating the Lagrangian diffusivity tensor for a finite time period $[0, \tau]$, this choice keeps the diffusivity estimates as local as possible.

The equilibration time $\tau_{\text {eq }}$ characterizes the time it takes for the particle velocity at $\tau$ to decorrelate from its velocity at $\tau=0$. We determine $\tau_{\text {eq }}$ as follows: The value of $\kappa_{i j}(\mathbf{x}, \tau)$ changes as $\tau$ increases and then it becomes insensitive to $\tau$, when the particle velocity at $\tau$ decorrelates from its velocity at $\tau=0$. This leveling off is typically termed the convergence of $\kappa_{i j}(\mathbf{x}, \tau)$. The detailed criterion to judge whether $\kappa_{i j}(\mathbf{x}, \tau)$ has converged at $\tau \in\left[\tau_{1}, \tau_{2}\right]$ is provided in the appendix of Chen et al. (2014b). In brief, we choose $\tau_{2}=\tau_{1}+30$ ndtus and if the rate of change of $\kappa_{i j}(\mathbf{x}, \tau)$ over $\tau \in\left[\tau_{1}, \tau_{2}\right]$ through a least squares fit is smaller than the minimum value of $2 \tilde{\sigma}(\tau)$ over $\tau \in\left[\tau_{1}, \tau_{2}\right]$, we consider that $\kappa_{i j}(\mathbf{x}, \tau)$ is converged at $\tau=\left(\tau_{1}+\tau_{2}\right) / 2$. The term $\tilde{\sigma}(\tau)$ is the error bar of $\kappa_{i j}(\mathbf{x}, \tau)$ at the $95 \%$ confidence level determined from the bootstrapping technique (Chen et al. 2014b). The smallest $\left(\tau_{1}+\tau_{2}\right) / 2$ with converged $\kappa_{i j}(\mathbf{x}, \tau)$ is termed as the equilibration time for $\kappa_{i j}(\mathbf{x}, \tau)$, that is, $\tau_{\mathrm{eq}}$. 

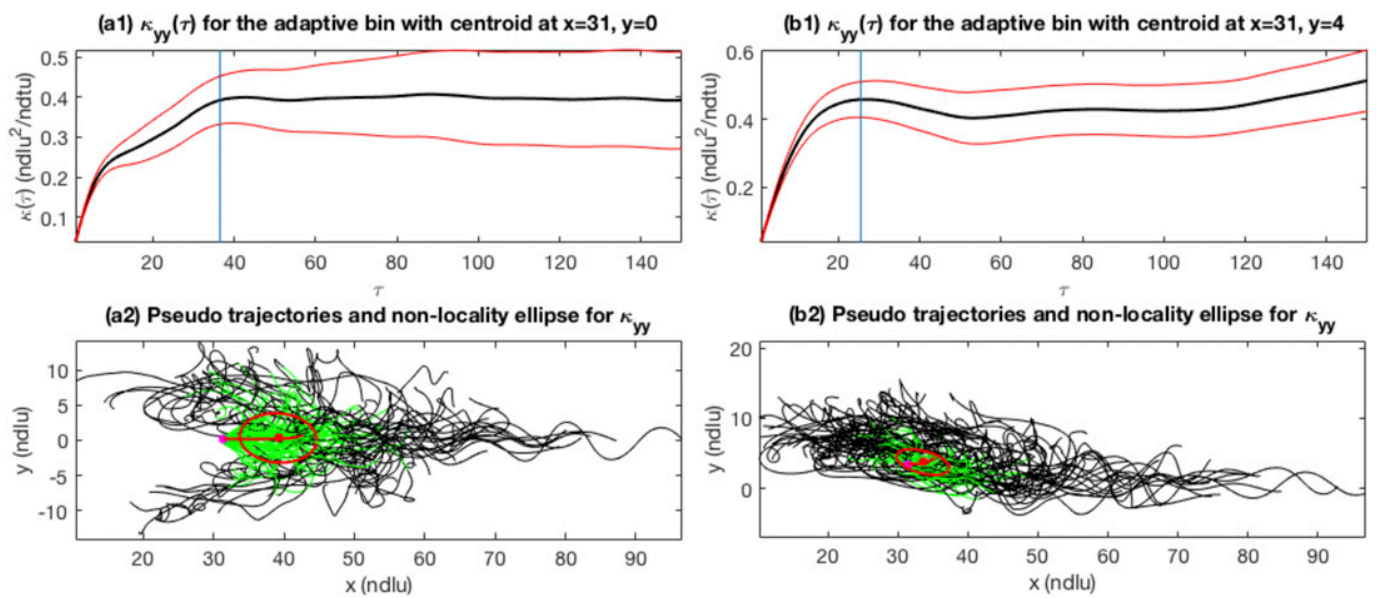

FIG. 4. Illustrations of the concepts of equilibration time, effective trajectories, nonlocality ellipses, and remapping. (a1) The diffusivity tensor component $\kappa_{y y}(\tau)$ (black) and its error bar at $95 \%$ confidence level (red) for the adaptive bin with centroid locating at $x=31$ ndlus, $y=0$ ndlu. The vertical line in blue indicates the equilibration time $\tau_{\text {eq. }}$ (a2). Trajectories of pseudoparticles originating from the adaptive bin, whose centroid is indicated by the magenta dot. Only $5 \%$ of trajectories are displayed here to make these trajectories visible. The portion of the effective trajectories corresponding to $\tau<\tau_{\text {eq }}$ are colored green, and the portion corresponding to $\tau>\tau_{\text {eq }}$ are colored black. The red ellipse is the nonlocality ellipse inferred from these effective trajectories. The ellipse center, indicated by the red dot, is the so-called track centroid. After diagnosing $\kappa_{y y}^{\infty}$ from $\kappa_{y y}(\tau)$, we remap its value from the bin centroid (magenta dot) to the track centroid (red dot). The red line denotes the Lagrangian mean trajectory estimated from all the pseudotrajectories, defined by Eq. (5). (b1), (b2) As in (a1) and (a2), but for the adaptive bin with the centroid locating at $x=31$ ndlus, $y=4$ ndlus.

Take Fig. 4 as an example: $\kappa_{y y}$ starts leveling off at $\tau=\tau_{\text {eq }}$, indicated by the blue vertical lines in Figs. 4a1 and $4 \mathrm{~b} 1$. In some cases, $\kappa_{i j}(\tau)$ only levels off for a limited time period. For example, in Fig. 4b1, the value of $\kappa_{y y}(\tau)$ increases at $\tau \in\left[0, \tau_{\text {eq }}\right]$ and equilibrates at $\tau \in[40,100]$ ndtus and then starts increasing again at $\tau>100$ ndtus. Therefore, diffusivities based on $\tau_{\text {eq }}$ can be considered to be the as local as possible diffusivity estimates.

\section{b. Estimating the nonlocality of mixing}

\section{1) Nonlocality ELLIPSES}

As stated in section 1, Lagrangian eddy diffusivities are inherently nonlocal, as diagnosing $\kappa_{i j}^{\infty}$ involves integrating a Lagrangian autocorrelation function over a finite time period and pseudotrajectories generally drift away from the bin centroid as $\tau$ increases. The Lagrangian mean trajectory, which refers to the trajectory of the centroid of the pseudotrajectories from the corresponding adaptive bin, is an elementary way to illustrate mixing nonlocality. It can be calculated from

$$
x_{L}(\tau)=\frac{\sum_{i=1}^{N} x_{i}(\tau)}{N}, \quad y_{L}(\tau)=\frac{\sum_{i=1}^{N} y_{i}(\tau)}{N},
$$

where $x_{L}(\tau)$ and $y_{L}(\tau)$ denote the Lagrangian mean trajectory. The terms $x_{i}(\tau)$ and $y_{i}(\tau)$ denote the zonal and meridional positions of the $i_{\text {th }}$ pseudotrajectory at the lead-lag time $\tau$ for a specific adaptive bin. Figure 5 shows the Lagrangian mean trajectories for each adaptive bin from $\tau=0$ to $\tau=60$ ndtus. These trajectories starting from the intense jet and recirculation gyres extend a long distance because of the strong mean flow there, but those starting from the wave radiation region, where the mean flow is weak, are generally short (Fig. 5). Although these Lagrangian mean trajectories tend to largely follow the mean streamline, the deviation from the streamline is also nonnegligible. This is unsurprising since the Lagrangian and Eulerian mean flow generally differ from each other.

Note that the value of $\kappa_{i j}^{\infty}$ is a function of the net characterization of a large number of pseudotrajectories from $\tau=0$ to $\tau=\tau_{\text {eq }}$, which collectively are termed the effective trajectories for a specific adaptive bin. These effective trajectories all have starting points within the adaptive bin but can cover a region with significant area, at times larger than the adaptive bin size. For example, consider the green tracks in Figs. $4 \mathrm{a} 2$ and $4 \mathrm{~b} 2$, which show the effective trajectories for the adaptive bin centered at the magenta dot. The particles are strongly advected by the intense jet flow and pass over a large area before $\kappa_{y y}$ converges.

Here, we introduce the concept of nonlocality ellipses to quantify the degree of mixing nonlocality for a diffusivity estimate computed from the effective trajectories in a given bin, for example, the trajectories in green 


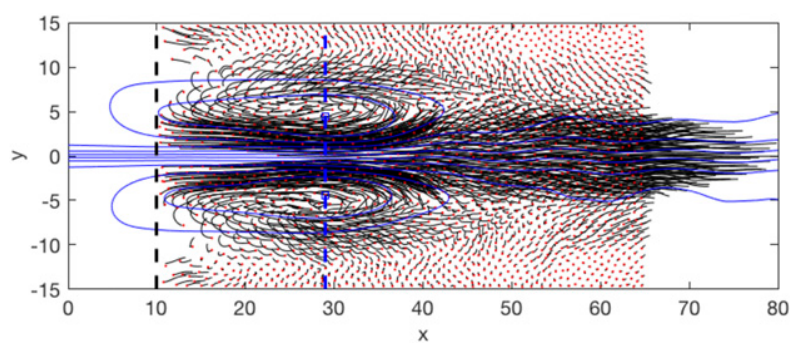

FIG. 5. Lagrangian mean trajectories from $\tau=0$ to $\tau=60$ ndtus, inferred from all the pseudotrajectories at each adaptive bin. The red points denote the centroids of the adaptive bins, that is, the starting points of each Lagrangian mean trajectory. Annotations denoting key features of the time-mean circulation and EKE distribution are as in Fig. 1.

for the adaptive bin at the magenta dot in Figs. $4 \mathrm{a} 2$ and $4 \mathrm{~b} 2$. The mean value of $x_{i}(\tau)$ and $y_{i}(\tau)$ over all the effective trajectories for a bin is defined as the centroid of these effective trajectories, that is, the track centroid $\left(x_{c}, y_{c}\right)$, and is computed as

$$
\begin{aligned}
& x_{c}=\frac{\int_{0}^{\tau_{\mathrm{eq}}} \sum_{i=1}^{N} x_{i}(t) d t}{N \tau_{\mathrm{eq}}}=\frac{\int_{0}^{\tau_{\mathrm{eq}}} x_{L}(\tau)}{\tau_{\mathrm{eq}}}, \\
& y_{c}=\frac{\int_{0}^{\tau_{\mathrm{eq}}} \sum_{i=1}^{N} y_{i}(t) d t}{N \tau_{\mathrm{eq}}}=\frac{\int_{0}^{\tau_{\mathrm{eq}}} y_{L}(\tau)}{\tau_{\mathrm{eq}}} .
\end{aligned}
$$

As in Chen et al. (2014b), we can then respectively define the variance of the zonal and meridional positions of all the effective trajectories relative to the track centroid:

$$
\begin{gathered}
\sigma_{x}^{2}=\frac{\int_{0}^{\tau_{e q}} \sum_{i=1}^{N}\left[x_{i}(t)-x_{c}(t)\right]^{2} d t}{N \tau_{e q}}, \\
\sigma_{y}^{2}=\frac{\int_{0}^{\tau_{e q}} \sum_{i=1}^{N}\left[y_{i}(t)-y_{c}(t)\right]^{2} d t}{N \tau_{e q}} .
\end{gathered}
$$

Consistently, the cross variance of these particle positions relative to the track centroid is

$$
\sigma_{x y}^{2}=\frac{\int_{0}^{\tau_{\mathrm{eq}}} \sum_{i=1}^{N}\left[x_{i}(t)-x_{c}(t)\right]\left[y_{i}(t)-y_{c}(t)\right] d t}{N \tau_{\mathrm{eq}}} .
$$

Finally, following Preisendorfer (1988), Morrow et al. (1994), and Chen et al. (2014b), we can respectively infer the variance of particle positions along the major and minor axes of a nonlocality ellipse, $\sigma_{1}^{2}$ and $\sigma_{2}^{2}$, from $\sigma_{x}^{2}, \sigma_{y}^{2}$, and $\sigma_{x y}^{2}$ :

$$
\begin{aligned}
& \sigma_{1}^{2}=\frac{1}{2}\left[\sigma_{x}^{2}+\sigma_{y}^{2}+\sqrt{\left(\sigma_{x}^{2}-\sigma_{y}^{2}\right)^{2}+4\left(\sigma_{x y}^{2}\right)^{2}}\right], \\
& \sigma_{2}^{2}=\left(\sigma_{x}^{2}+\sigma_{y}^{2}\right)-\sigma_{1}^{2} .
\end{aligned}
$$

Collectively, the relationship between these variances can be visualized in the form of an ellipse with semimajor and semiminor axes $\sqrt{\sigma_{1}^{2}}$ and $\sqrt{\sigma_{2}^{2}}$ and an orientation of the major axis measured anticlockwise from the positive $x$ direction $\theta$ diagnosed from

$$
\tan (\theta)=\frac{\sigma_{1}^{2}-\sigma_{x}^{2}}{\sigma_{x y}^{2}}
$$

In this way, mixing nonlocality can be described by three variables characterizing ellipse geometry: ellipse area, eccentricity, and tilt. The ellipse area $\pi \sqrt{\sigma_{1}^{2}} \sqrt{\sigma_{2}^{2}}$ quantifies the bulk degree of mixing nonlocality, the ellipse eccentricity $\left(\sigma_{1}^{2}-\sigma_{2}^{2}\right) /\left(\sigma_{1}^{2}+\sigma_{2}^{2}\right)$ quantifies the anisotropy of the mixing nonlocality in a principal axes frame, and the ellipse tilt $\theta$ describes the direction along which this anisotropy is oriented.

As an example, we illustrate the nonlocality ellipse for the adaptive bin with the centroid at the magenta dot in Figs. $4 \mathrm{a} 2$ and $4 \mathrm{~b} 2$. We place the center of the ellipse at the track centroid, indicated by the red dot, rather than at the bin centroid, indicated by the magenta dot, as the nonlocality ellipse is best interpreted as representing the variance of particle positions relative to the track centroid. Our analysis indicates that, in this idealized jet model, the shape and orientation of the nonlocality ellipses are to a large extent similar among the four $\kappa_{i j}$ components (section 4). On the other hand, since nonlocality ellipses strongly depend on the equilibration time [Eqs. (6)-(10)], and because this differs among the four $\kappa_{i j}$ components, the size of the four nonlocality ellipses for the four $\kappa_{i j}$ components at a given location can be quite different (see section 4 for details).

\section{2) Mixing ESTIMATE REMAPPING}

The recognition that the track centroid, as opposed to the bin centroid, is a better representation of the spatial location of the diffusivity estimate, motivates a remapping of our diffusivity estimates. Consider the following: each adaptive bin contains the starting point of a number of pseudotrajectories and thus corresponds to a distinct estimate of the diffusivity tensor $\kappa_{i j}^{\infty}$. This estimate, however, is a representation of the mixing characteristics in the area covered by the entire span of these effective trajectories (a function of the equilibration time of $\kappa_{i j}^{\infty}$ and the particle advecting velocity), which can be significantly larger and significantly shifted from the bin centroid characterizing the trajectories' starting points within the adaptive bin. As such, it is more 
reasonable to assign the obtained diffusivity value to the center of the nonlocality ellipse, that is, the track centroid defined by Eq. (6), which characterizes the span of the effective trajectories, as opposed to the bin centroid, which characterizes where the effective trajectories originate. An example of the impact of this remapping is shown in Fig. 4, where we remap $\kappa_{y y}^{\infty}$ for the selected adaptive bin from the bin centroid (magenta dot) to the track centroid (red dot).

The distance between the track centroid and bin centroid itself becomes its own metric of the nonlocality of mixing: this is visualized for $\kappa_{x x}^{\infty}$ in the idealized WBC jet model in Fig. 6a. Note that, considering that particles are advected by the total flow, which includes both mean flow and eddies, the nonlocality properties depend on both the mean and eddy characteristics. Owing to the strong mean flow inside the jet, here the nonlocality ellipses can be largely due to the mean flow contribution and as such do not necessarily quantify large eddy mixing in this case. Here, we see that this distance is largest within the intense jet, where it approaches a maximum value of the same order as the distance between the inflow and the downstream location of jet stabilization for this particular set of inflowing jet parameters. This indicates significant nonlocality of mixing here. Because of significant distances in key regions, the spatial pattern of $\kappa_{x x}^{\infty}$ mapped onto the track centroid differs from that mapped onto the bin centroid (Figs. 6b,c) in some important ways. For example, after remapping the diffusivity estimates from the bin to the track centroids, the two distinct regions of elevated $\kappa_{x x}^{\infty}$ inside the jet, centered around $x=15$ and $x=35$, respectively, are both shifted eastward, a consequence of the intense eastward advection of the jet. This eastward shifting of mixing structures within the jet caused by remapping also occurs for the other three components of $\kappa_{i j}^{\infty}$ (not shown). In contrast, the spatial structure of mixing structures within the recirculation gyres does not change significantly, as the flow there is weaker. The distance between the track centroid and bin centroid has large spatial variability and thus remapping has a spatially varying impact on the pattern of mixing structures.

\section{c. Estimating the anisotropy of mixing from mixing ellipses}

We expect that the anisotropy inherent in zonally elongated, highly anisotropic systems like jets can lead to asymmetries and spatial heterogeneity not only in the nonlocality of mixing, but also in the mixing rate itself. These asymmetries and heterogeneities could manifest themselves in a number of ways: significant differences in the along-jet versus cross-jet components
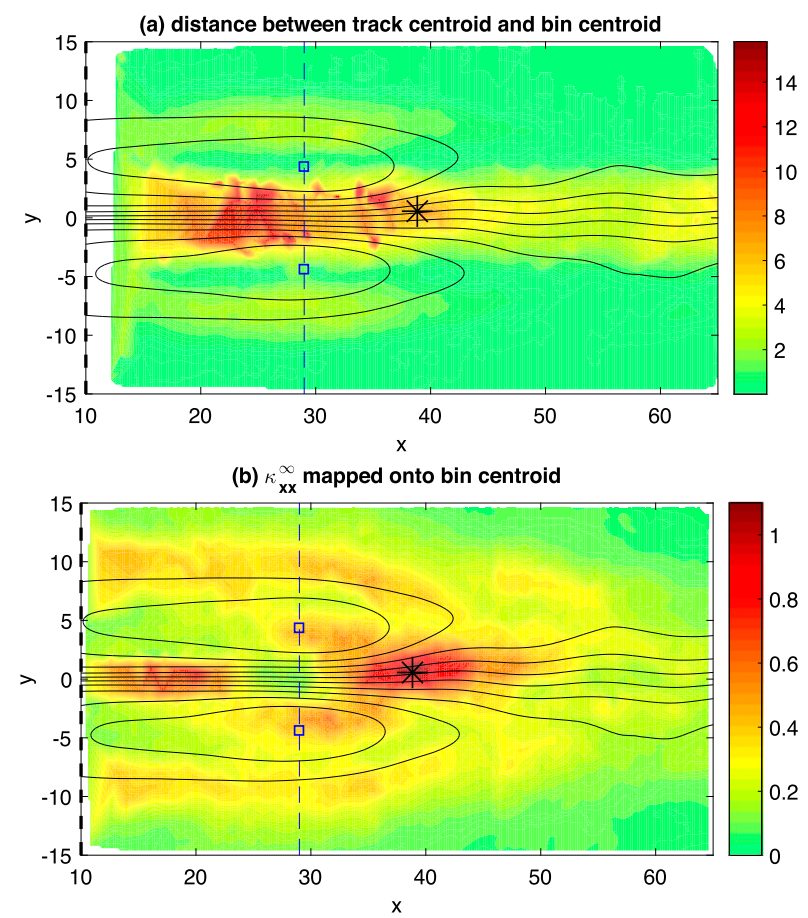

(c) $\kappa_{\mathbf{x x}}^{\infty}$ mapped onto track centroid

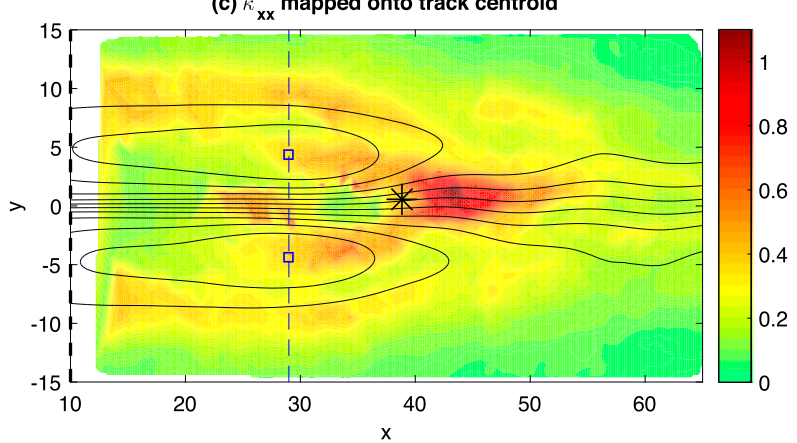

FIG. 6. (a) The distance between the track centroid and the corresponding adaptive bin centroid in the idealized WBC jet model and a comparison of the spatial pattern of $\kappa_{x x}^{\infty}$ mapped onto (b) the adaptive bin centroid and (c) the track centroid determined from Eq. (6). Results here are those interpolated from either the adaptive bin or track centroids onto regular grids. No estimates are provided in the western sponge layer $(0<x<10)$ because of artificial damping there. Annotations denoting key features of the time-mean circulation and EKE distribution are as in Fig. 1.

of the mixing rate, asymmetries in the various components of the full diffusivity tensor more generally, and/or significant spatial variability in the magnitude and anisotropy of mixing rates, for example. In this study we employ mixing ellipses, described below, to quantify the dominant magnitude, anisotropy, and direction of eddy mixing rates and their patterns in space in order to characterize the anisotropy of mixing in this system. 
(a) non-locality ellipse for $\kappa_{x x}^{\infty}$

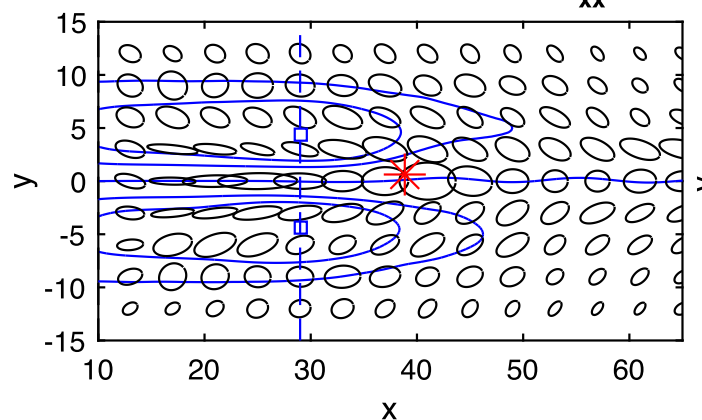

(c) non-locality ellipse for $\kappa_{\mathbf{y x}}^{\infty}$

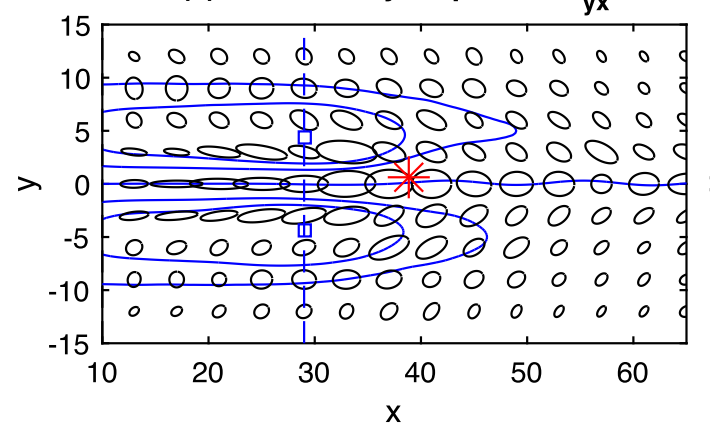

(b) non-locality ellipse for $\kappa_{\mathrm{yy}}^{\infty}$

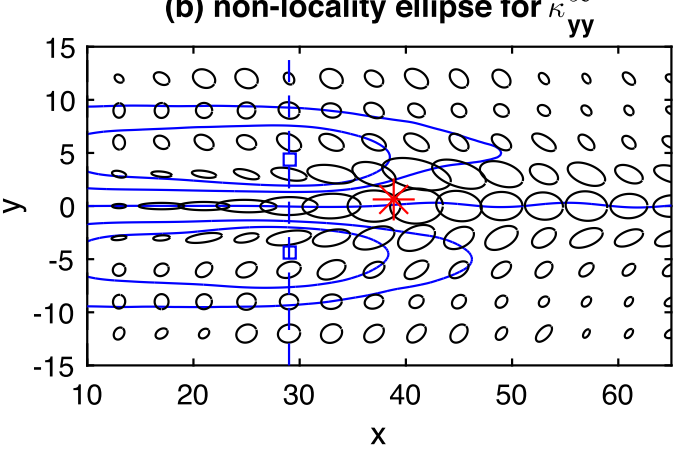

(d) non-locality ellipse for $\kappa_{\mathbf{x y}}^{\infty}$

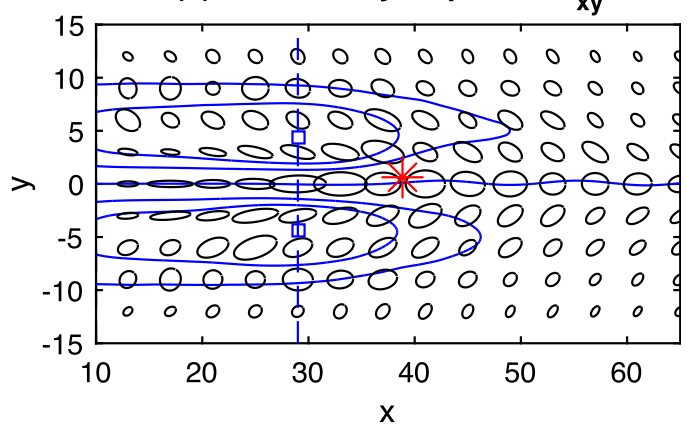

FIG. 7. Nonlocality ellipses for $\kappa_{i j}^{\infty}$. Ellipses are displayed at a resolution of 4 ndlus in the $x$ direction and 3 ndlus in the $y$ direction, and the length of the major and minor axes of ellipses are scaled to be half of the actual values with units of ndlus. The choice of plotting resolution and scaling is a compromise between showing detailed spatial structures and making the ellipses visible. Annotations denoting key features of the time-mean circulation and EKE distribution are as in Fig. 1.

As a second rank tensor, the diffusivity tensor $\kappa_{i j}^{\infty}$ can be uniquely decomposed into the sum of a symmetric part $S_{i j}$ and an antisymmetric part $i j$ :

$$
\kappa_{i j}^{\infty}=\underbrace{\left(\kappa_{i j}^{\infty}+\kappa_{j i}^{\infty}\right) / 2}_{S_{i j}}+\underbrace{\left(\kappa_{i j}^{\infty}-\kappa_{j i}^{\infty}\right) / 2}_{A_{i j}} .
$$

Physically, this decomposition separates the along-gradient component of eddy mixing described by the symmetric diffusivity tensor (the so-called diffusive flux) from the cross-gradient component described by the antisymmetric diffusivity tensor (the so-called skew flux, equivalent to an advection by some divergence-free velocity; see, e.g., Vallis 2006). Given the skew diffusion has no effect on along-gradient fluxes, and hence the irreversible mixing of a tracer, we characterize the anisotropy of mixing via mixing ellipses based on properties of $S_{i j}$ only.

Assuming the four components of $\kappa_{i j}^{\infty}$ have the same nonlocality property, which qualitatively holds in this idealized model (Figs. 7, 8), we can estimate mixing ellipses as follows: First, we diagnose the diffusivity tensor $\kappa_{i j}^{\infty}$ from Eqs. (3) and (4) and then we estimate the symmetric part of $\kappa_{i j}^{\infty}$, that is, $S_{i j}$, from Eq. (11). Next, we define $\sigma_{x}^{2}=S_{x x}, \sigma_{y}^{2}=S_{y y}$, and $\sigma_{x y}^{2}=S_{x y}$, where $S_{x x}, S_{y y}$, and $S_{x y}$ are components of the symmetric tensor $S_{i j}$ in a defined $x-y$ coordinate system. Third, similar to our diagnosis of nonlocality ellipses, using $\sigma_{x}^{2}, \sigma_{y}^{2}$, and $\sigma_{x y}^{2}$, we obtain the variances in the principal axes directions and the orientation of the principal axes frame $\sigma_{1}^{2}, \sigma_{2}^{2}$, and $\theta$, respectively, from Eqs. (9) and (10). Note that, for nonlocality ellipses, we define their semimajor and semiminor axes' lengths as $\sqrt{\sigma_{1}^{2}}$ and $\sqrt{\sigma_{2}^{2}}$, respectively, and the unit is consistent with that of distance. Here, for mixing ellipses, we define the semimajor and semiminor axes' lengths as $\sigma_{1}^{2}$ and $\sigma_{2}^{2}$. This ensures that the axes lengths have the same units as $\kappa_{i j}^{\infty}$. Another reason for this choice is that $\sigma_{2}^{2}$ can be negative in the area with negative diffusivities and thus defining $\sqrt{\sigma_{2}^{2}}$ as the axis length would lead to imaginary values of axis length.

The visualization of mixing ellipses is a useful way to gain insight into the geometry of mixing and its spatial variability: ellipse size illustrates the magnitude of mixing rates, ellipse eccentricity reflects mixing rate anisotropy, and ellipse tilt indicates the dominant mixing direction. As a means of illustration, consider some simple examples. If the mixing rate in the zonal direction is large relative to those in other directions (i.e., $S_{x x}$ is 
(a) Histogram of semimajor axis

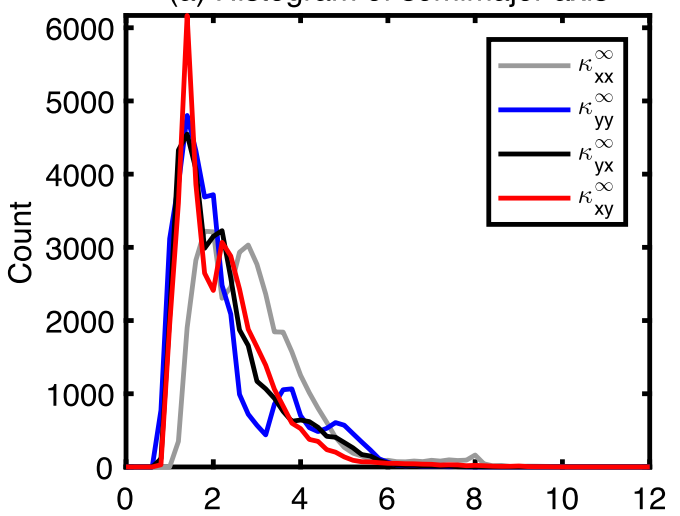

Semimajor axis of non-locality ellipse (ndlu)

(c) Histogram of eccentricty

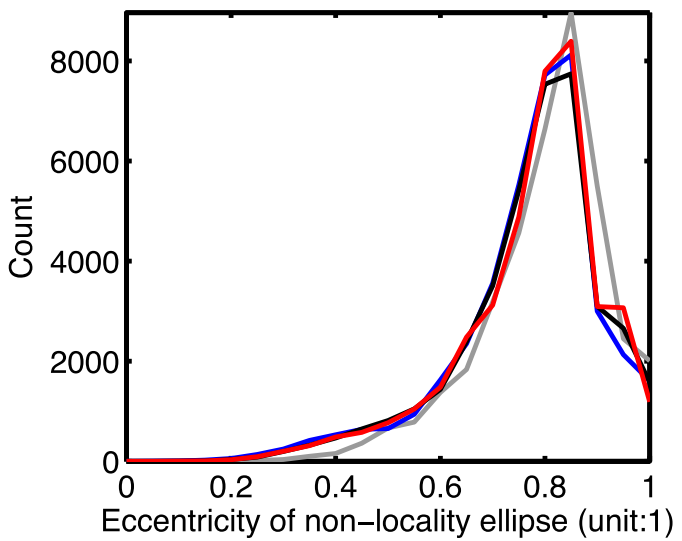

(b) Histogram of semiminor axis

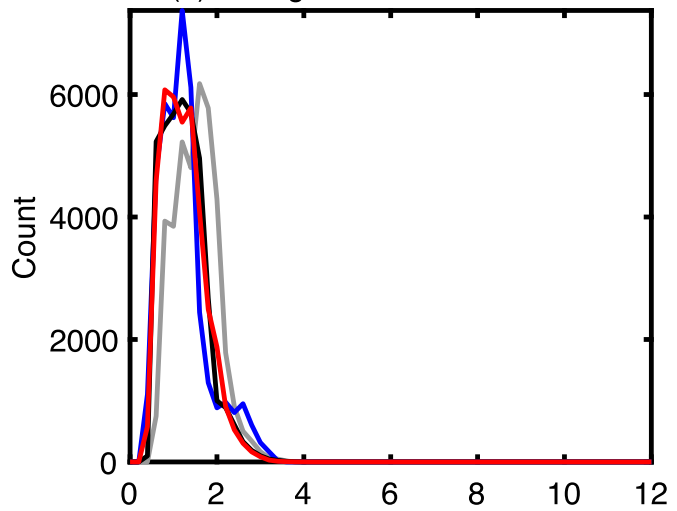

Semiminor axis of non-locality ellipse (ndlu)

(d) Histogram of tilt $(\theta)$

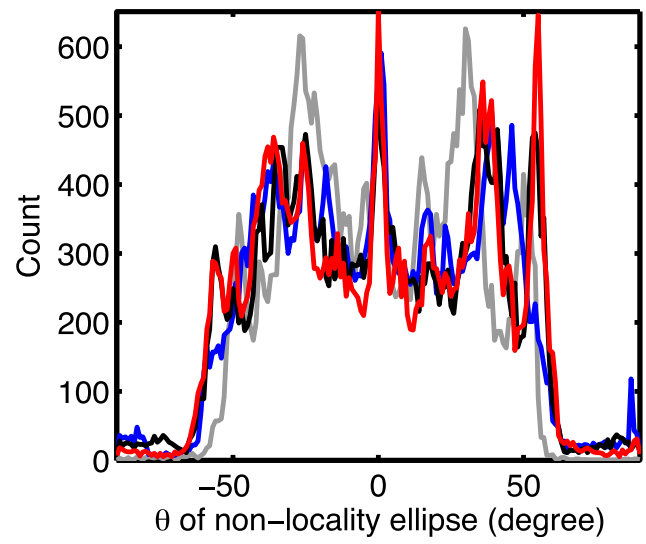

FIG. 8. Histograms of (a) semimajor axis length $\sqrt{\sigma_{1}^{2}}$, (b) semiminor axis length $\sqrt{\sigma_{2}^{2}}$, (c) eccentricity $\sqrt{1-\sigma_{2}^{2} / \sigma_{1}^{2}}$, and (d) tilt $\theta$ of nonlocality ellipses of $\kappa_{i j}^{\infty}$ mapped onto track centroids. The histograms in (a), (b), (c), and (d) are sorted in 0.2-ndlu, 0.2 -ndlu, $0.05^{\circ}$, and $1^{\circ}$ bins, respectively. The terms $\sigma_{1}^{2}, \sigma_{2}^{2}$, and $\theta$ are defined in Eqs. (9) and (10).

large relative to the other components of $S_{i j}$ ), the mixing ellipse is quasi zonal with a semimajor axis length approximately equal to $S_{x x}$ and a semiminor axis length approaching zero. If instead $S_{x x} \approx S_{y y}$ and $S_{x y} \approx 0$ (i.e., mixing is quasi isotropic), the mixing ellipse is quasi circular with semimajor and semiminor axis lengths equal to $S_{x x}$. Note that the mixing ellipse can also represent negative diffusivities. For example, if $S_{x x}$ and $S_{x y}$ are approximately zero, but $S_{y y}$ is negative, the mixing ellipse's semimajor axis is quasi zonal with length approaching zero, and its semiminor axis length is negative with a value of $S_{y y}$. In other words, here negative diffusivity manifests itself as a negative semiminor axis length.

\section{Results I: Mixing nonlocality}

\section{a. Nonlocality ellipses: Characteristics, spatial patterns, and similarity}

A visualization of the nonlocality ellipses for each of the four components of $\kappa_{i j}^{\infty}$ is shown in Fig. 7. To compute these ellipses, we first interpolate $\sigma_{x}^{2}, \sigma_{y}^{2}$, and $\sigma_{x y}^{2}$ at the track centroids onto a regular grid at 0.2-ndlu resolution and then use these values to diagnose the nonlocality ellipses at this same uniform spatial resolution. Figure 7 shows us clearly that the nonlocality ellipses have finite area everywhere in the domain, implying that mixing is nonlocal to some degree in all regions.

A second conclusion from this visualization is that there are several systematic, large-scale patterns in the distributions of nonlocality ellipse characteristics and further that these patterns are largely similar for all four $\kappa_{i j}^{\infty}$ components. For example, the nonlocality ellipses for each $\kappa_{i j}^{\infty}$ component are all relatively large and isotropic in the vicinity of the EKE maximum, a consequence of large values of both $\sigma_{x}^{2}$ and $\sigma_{y}^{2}$ for all four $\kappa_{i j}^{\infty}$ components (not shown). Further, across all $\kappa_{i j}^{\infty}$ components, the ellipses tend to be zonally elongated inside the jet in the upstream region (a consequence of extremely large values of $\sigma_{x}^{2}$ ). These results are consistent with the expectation given that in the upstream region the zonal jet 


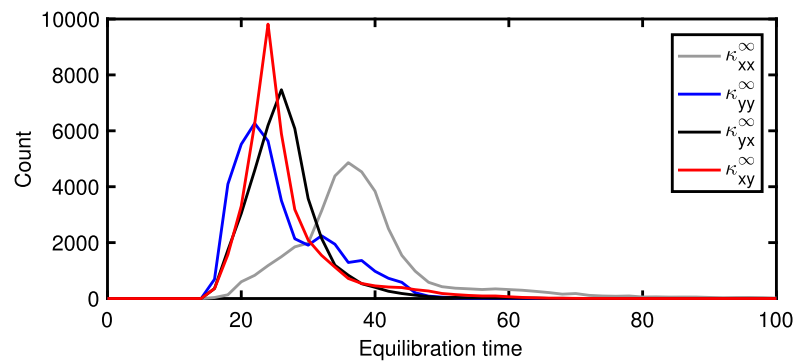

FIG. 9. Histogram of the equilibration time $\tau_{\mathrm{eq}}$ for $\kappa_{x x}^{\infty}, \kappa_{y y}^{\infty}, \kappa_{y x}^{\infty}$, and $\kappa_{x y}^{\infty}$ sorted in 2-ndtu bins.

flow is strong and, as a consequence, particles largely follow the jet and move eastward. In contrast, in the downstream region, the jet flow is relatively weak and the flow field is dominated by eddy motions, which are generated from the jet's instability upstream and become finite amplitude downstream. Thus, in the downstream region, particles move both zonally and meridionally. A third similarity among all four $\kappa_{i j}^{\infty}$ components is a largescale pattern of ellipse tilt in the vicinity of the jet in the upstream region, in which the ellipses tend to tilt toward the jet on the jet flanks (a consequence of $\sigma_{x y}^{2}$ having positive values south of the jet and negative values north of the jet). Note that this pattern is similar to that seen in the eddy covariance ellipses in this region, which is indicative of an eddy momentum flux consistent with eddies stabilizing the jet through a barotropic instability process (see Waterman and Lilly 2015). The relationship between metrics of mixing rate and anisotropy derived from Lagrangian statistics, as is the case here, and Eulerian statistics, as is the case for eddy covariance ellipses, is the subject of ongoing investigation.

The distribution of ellipse characteristics, as well as the degree of similarity in these distributions across all $\kappa_{i j}^{\infty}$ components, is visualized in histograms of ellipse semimajor and semiminor axis length, eccentricity, and tilt in Fig. 8. Here, the high degree of similarity in the distributions of ellipse characteristics across all $\kappa_{i j}^{\infty}$ components is further documented: the lengths of the semimajor axis and semiminor axis for all four $\kappa_{i j}^{\infty}$ components show similar-shaped distributions and range from 0 to 6 ndlus and from 0 to 4 ndlus, respectively (Figs. 8a,b); the ellipse eccentricity histograms for all $\kappa_{i j}^{\infty}$ components all have a single peak near 0.8 and typical values from 0.2 to 1 (Fig. 8c); and finally the distributions of ellipse tilt for all $\kappa_{i j}^{\infty}$ components typically range from $-50^{\circ}$ to $50^{\circ}$.

The nonlocality ellipses for $\kappa_{x x}^{\infty}$ do tend to be larger than the other three components of $\kappa_{i j}^{\infty}$ (Figs. 8a,b), consistent with the relatively large equilibration time for $\kappa_{x x}^{\infty}$ (see Fig. 9 and subsequent discussion). Peak values of the distributions of the semimajor and semiminor axis lengths for $\kappa_{x x}^{\infty}$, respectively occurring at 1.8 and 1.6

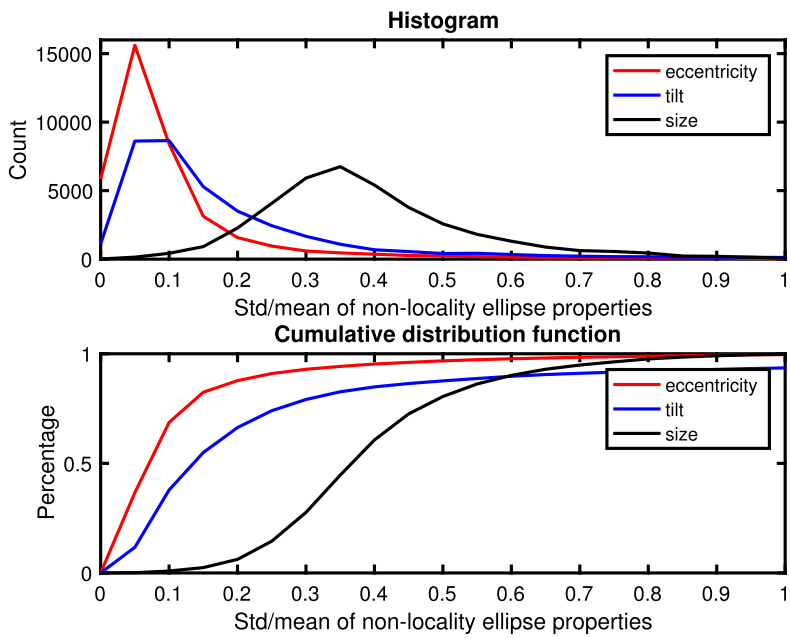

FIG. 10. (top) Histogram and (bottom) cumulative distribution function of the normalized variation of nonlocality ellipse properties (eccentricity, tilt, and size) in the study domain $x=10-65$ ndlus and $|y|<=15$ ndlus. At each location, there are four nonlocality ellipses, corresponding to different components of $\kappa_{i j}^{\infty}$. Thus, the standard deviation and mean of a certain ellipse property (e.g., tilt) at each location can be diagnosed using the four available values. The normalized variation is the ratio between the standard deviation and the mean.

ndlus, are larger than those for the other three $\kappa_{i j}^{\infty}$ components, which average 1.4 and 1.1 ndlus, respectively (Figs. 8a,b). Figures 7 and 8 thus indicate that at a given location, the four nonlocality ellipses, corresponding to the four components of $\kappa_{i j}^{\infty}$, are similar: their tilt and eccentricity are quasi similar, but their sizes differ. This is further seen via consideration of the standard deviation of the properties of the four ellipses divided by their mean value, a useful metric to quantify the amount of variability among the four nonlocality ellipses at each location (Fig. 10). The percentage of the eccentricity and tilt metrics with values smaller than 0.3 is approximately $70 \%$ and $90 \%$, respectively (note smaller values indicate more similarity among the ellipses for each $\kappa_{i j}^{\infty}$ component). In contrast, this percentage for ellipse size is only $\sim 25 \%$. These values suggest that the ellipse tilt and eccentricity typically vary little among the four ellipses at each location, however, the ellipse size can vary significantly.

\section{b. Interpretation}

\section{1) ON THE SIZES OF NONLOCALITY ELLIPSES}

The properties of nonlocality ellipses at a given location for different components of $\kappa_{i j}^{\infty}$ vary because they are inferred from the effective trajectories. These in turn vary across $\kappa_{i j}^{\infty}$ components because they depend on the equilibration time that, owing to anisotropy in the jet 

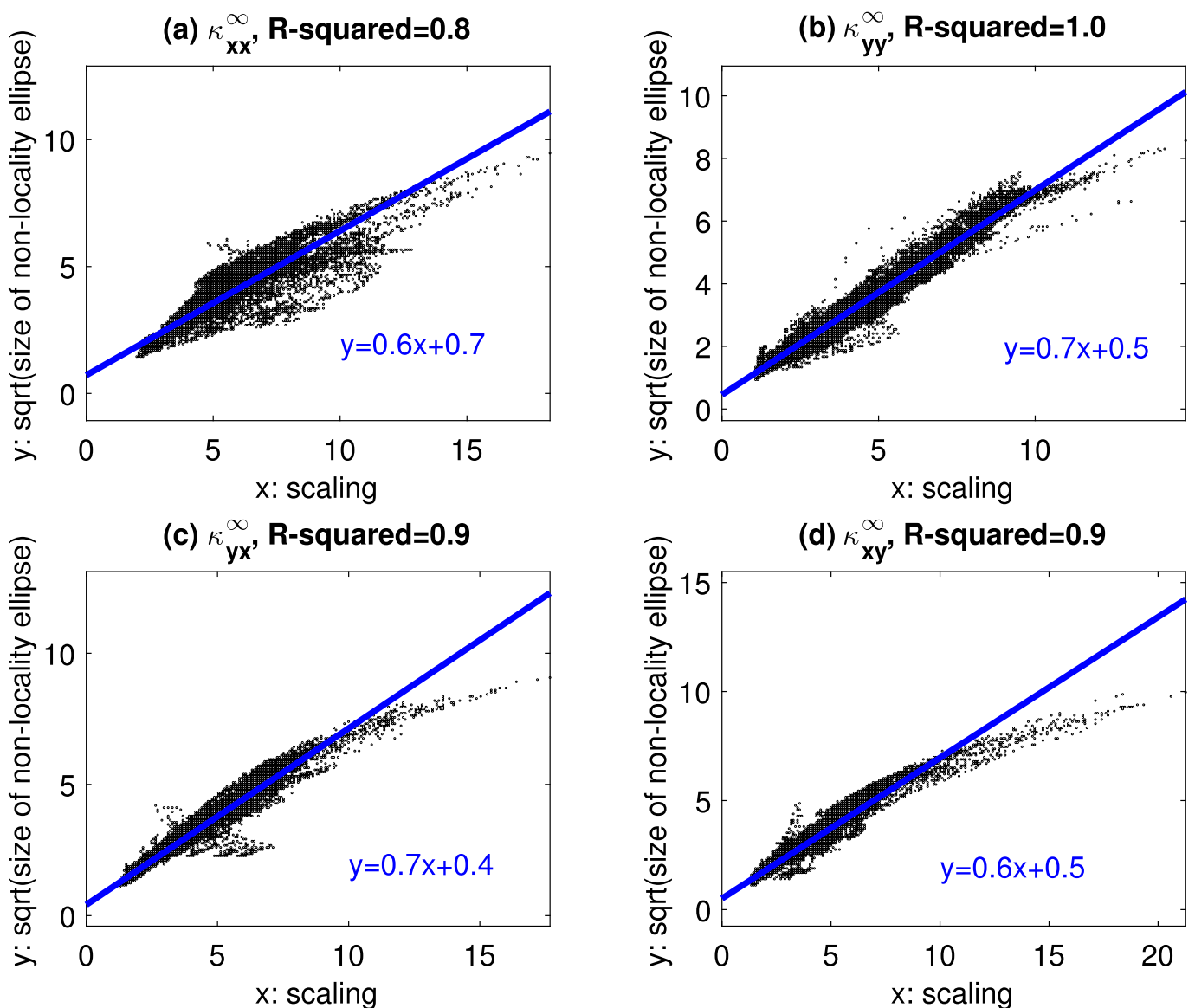

FIG. 11. Scatterplots between the scaling $\sqrt{\pi\left[\left(\tau_{\text {eq }} V_{\text {eddy }}\right) / 2\right]^{2}}$ ( $x$ axis; ndlu $)$ and the observed square root of the size of the nonlocality ellipse ( $y$ axis, ndlu) for $(\mathrm{a}) \kappa_{x x}^{\infty}$, (b) $\kappa_{y y}^{\infty}$, (c) $\kappa_{y x}^{\infty}$, and (d) $\kappa_{x y}^{\infty}$. Blue lines indicate the best linear least squares fit, and the blue text shows the corresponding function.

flow and eddy properties, varies for different $\kappa_{i j}^{\infty}$ components. Figure 9 shows this variability via histograms of the equilibration time for each $\kappa_{i j}^{\infty}$ component. Values of the equilibration time range from 10 to 80 ndtus. The equilibration time corresponding to the peak value of the histogram is 36 ndtus for $\kappa_{x x}^{\infty}, 22$ ndtus for $\kappa_{y y}^{\infty}, 26$ ndtus for $\kappa_{y x}^{\infty}$, and 24 ndtus for $\kappa_{x y}^{\infty}$. Thus, we expect $\kappa_{x x}^{\infty}$ to typically have a significantly larger characteristic equilibration time compared to the other three components. As a consequence, we expect the effective trajectories for $\kappa_{x x}^{\infty}$ to typically cover a larger area than those for the other three $\kappa_{i j}^{\infty}$ components, and, correspondingly, we also expect the nonlocality ellipses for this component to be larger, as is indeed seen in the model output.

We seek a more quantitative understanding of the controls on nonlocality ellipse size and use the model output to assess the validity of a hypothesized scaling. Given that the area of the nonlocality ellipse represents the spreading area of the particle trajectories within the equilibration time $\tau_{\text {eq }}$ and since the particles are advected by a flow field dominated by eddy motions, we assume that the length of the effective trajectories scales as $\tau_{\text {eq }}$ times $V_{\text {eddy }}$ and thus that the axis length of the nonlocality ellipses scales with $\tau_{\text {eq }} V_{\text {eddy }} / 2$, where $V_{\text {eddy }}$ is the time-mean eddy velocity magnitude. If valid, we can then express the square root of the nonlocality ellipse area, a useful metric of the degree of mixing nonlocality, as a function of $\tau_{\mathrm{eq}} V_{\text {eddy }}$ as follows:

$\sqrt{\text { non - locality ellipse area }} \approx \alpha \sqrt{\pi\left[\left(\tau_{\text {eq }} V_{\text {eddy }}\right) / 2\right]^{2}}+\beta$

If particle trajectories were straight lines (and particles were advected only by eddies), we would expect $\alpha \approx 1$ and $\beta \approx 0$. However, the convoluted structure of particle trajectories (Fig. 4), arising from the complex spatialtemporal variability of the turbulent flow, suggests that $\alpha$ is likely to be smaller than one.

Scatterplots testing this scaling for the jet model output are shown in Fig. 11. Here, we see that the degree 
TABLE 1. $R$-squared values characterizing the relationship between nonlocality ellipse and momentum ellipse eccentricity and tilt. The second column shows the $R$-squared values diagnosed from all points of the study domain. The third column shows those diagnosed from the points with a large degree of mixing nonlocality (size of nonlocality ellipse larger than $10^{0.7} \mathrm{ndlu}^{2}$ ). The $R$-squared values diagnosed from the remaining points (those with a small degree of mixing nonlocality) are shown in the fourth column. Here, the size, eccentricity, and tilt of nonlocality ellipses denote those averaged over four nonlocality ellipses at each location.

\begin{tabular}{lccc}
\hline \hline \multicolumn{1}{c}{ Pair } & $\begin{array}{c}R \text {-squared } \\
\text { from all points }\end{array}$ & $\begin{array}{c}R \text {-squared from large } \\
\text { nonlocality points }\end{array}$ & $\begin{array}{c}R \text {-squared from small } \\
\text { nonlocality points }\end{array}$ \\
\hline Eccentricity: Nonlocality vs full momentum ellipses & 0.1 & 0.1 & 0.5 \\
Tilt: Nonlocality vs full momentum ellipses & 0.7 & 0.6 & 1.0 \\
Eccentricity: Nonlocality vs eddy momentum ellipses & 0.0 & 0.0 & 0.5 \\
Tilt: Nonlocality vs eddy momentum ellipses & 0.6 & 0.4 & 1.0 \\
\hline
\end{tabular}

of nonlocality indeed increases with the increase of $\sqrt{\pi\left[\left(\tau_{\text {eq }} V_{\text {eddy }}\right) / 2\right]^{2}}$ and the corresponding $R$-squared values, which denote the percentage of the observed values explained by the linear scaling function [Eq. (12)] range from 0.8 to 1.0. These high $R$-squared values indicate that the linear empirical model [Eq. (12)] is a reasonable representation of the degree of mixing nonlocality. To assess how well the value of $\sqrt{\pi\left[\left(\tau_{\text {eq }} V_{\text {eddy }}\right) / 2\right]^{2}}$ captures the magnitude of $\sqrt{\text { non - locality ellipse area }}$, we estimate the values $\alpha$ and $\beta$ from the model data using a least squares fit. The variable $\alpha$ for the four $\kappa_{i j}$ components ranges from 0.6 to 0.7 and $\beta$ ranges from 0.4 to 0.7 ndlus. If we replace $V_{\text {eddy }}$ by the total flow magnitude in the scaling, $\alpha$ ranges from 0.1 to 0.2 and $\beta$ ranges from 1.7 to 2.8 .

\section{2) ON THE ECCENTRICITY AND TILT OF NONLOCALITY ELLIPSES}

We also seek a better understanding of the controls on nonlocality ellipse shape and orientation. As noted previously, we observe a suggestive similarity in the patterns of nonlocality ellipse eccentricity and tilt on the jet flanks with those of the eddy covariance ellipses discussed in Waterman and Lilly (2015). Eddy covariance ellipses are based on the temporally averaged Eulerian eddy momentum fluxes, while the nonlocality ellipses result from the dispersion of Lagrangian particles within the equilibration time. Similarities between these ellipses thus indicate a possible link between Eulerian and Lagrangian quantities.

Motivated by this idea, we compare the tilt and eccentricity of nonlocality ellipses to the tilt and eccentricity of eddy, as well as total flow (mean + eddy), covariance ellipses (hereafter eddy and full momentum ellipses, respectively) in the entire model domain. Details about diagnosing the tilt and eccentricity of these momentum ellipses are provided in appendix A. The $R$-squared values to quantify the degree of correspondence between these ellipse properties are given in Table 1, and scatterplots diagramming the relation between the eccentricity of the nonlocality ellipse with that of the total flow momentum ellipse at each point as a function on nonlocality is shown in Fig. 12. These analyses suggest that there is a reasonable degree of correspondence between nonlocality ellipse and momentum ellipse tilt ( $R$-squared values of 0.7 and 0.6 for full and eddy momentum ellipses, respectively) but, in general, a poor correspondence between nonlocality ellipse and momentum ellipse eccentricity $(R$-squared values of 0.1 and 0.0 for full and eddy momentum ellipses, respectively). Figure 12 suggests, however, that the degree of correspondence is a function of the degree of mixing

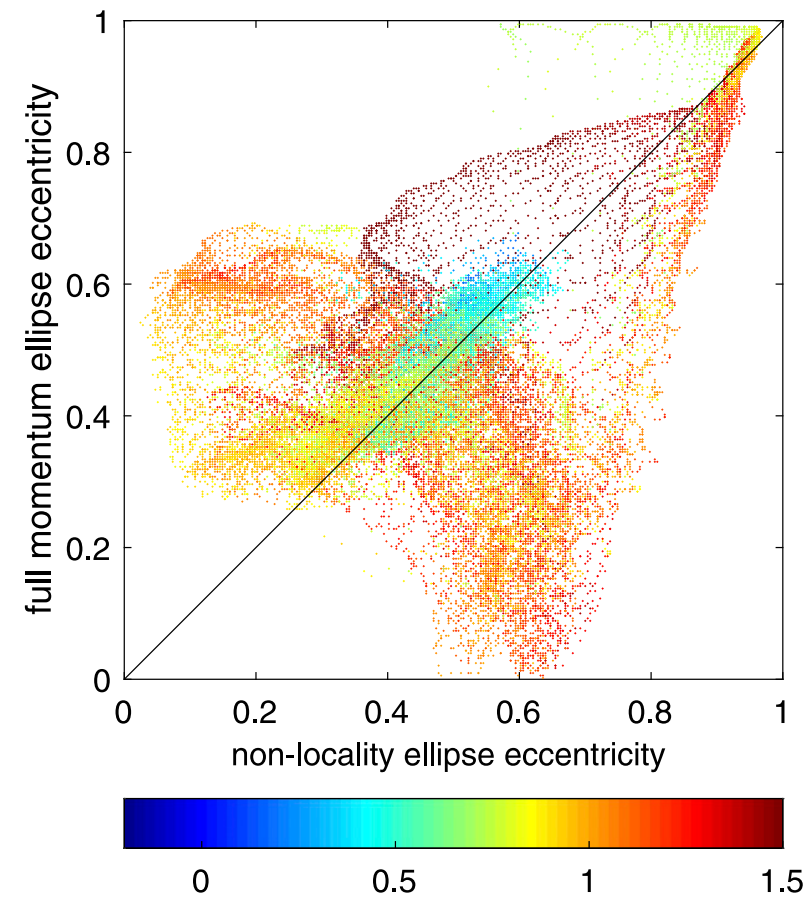

FIG. 12. Scatterplot illustrating the relation between the eccentricity of the nonlocality ellipse and the corresponding full momentum ellipse. Color indicates the size of the nonlocality ellipse on a logarithmic scale, a measure of the degree of mixing nonlocality at that point. The black line shows a 1:1 relation. Here, the size and eccentricity of the nonlocality ellipses correspond to the average of the size and eccentricity of four nonlocality ellipses at each location. 


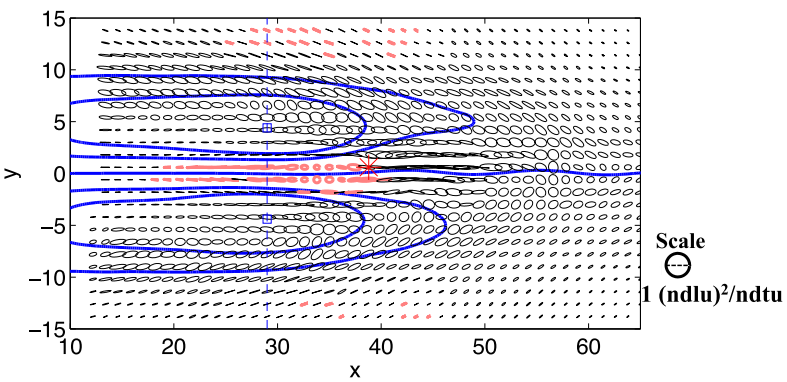

FIG. 13. Mixing ellipses inferred from the symmetric part of the diffusivity tensor. Black ellipses are those with semimajor and semiminor axis lengths both positive. Ellipses in red are those with positive major axis length but negative minor axis length, implying a negative diffusivity in the direction of the semiminor axis. Ellipses are plotted at a resolution of $1.2 \mathrm{ndlu} \times 1.2 \mathrm{ndlu}$. Annotations denoting key features of the time-mean circulation and EKE distribution are as in Fig. 1.

nonlocality itself, and indeed all $R$-squared values are significantly improved if only locations where the degree of nonlocality (here measured by the area of the nonlocality ellipse) is small (see Table 1). These analyses further show that mixing nonlocality ellipse properties are more closely matched to the properties of the full momentum ellipses compared to those of ellipses derived from the eddy momentum fluxes only. This is consistent with the expectation that it is the total flow (and not just the eddy component) that is important for the dispersion of Lagrangian particles and thus defining the degree of mixing nonlocality. An enhancement of the Lagrangian dispersion by the mean flow shear probably contributes to the relation between Eulerian total flow momentum ellipses and the Lagrangian nonlocality ellipses.

\section{Results II: Mixing anisotropy}

\section{a. Mixing ellipses: Characteristics and spatial patterns}

Figure 13 presents the mixing ellipses for the WBC jet system calculated following the method outlined in section 3c. The values of $S_{i j}$ used for mixing ellipse diagnosis are those mapped onto track centroids and then interpolated to a regular grid at 0.2-ndlu resolution. When interpreting Fig. 13, one should also take into account the mixing nonlocality information from Figs. 7 and 8 , as mixing ellipses are nonlocal and represent mixing in an area characterized by the nonlocality ellipse.

As was the case for the distribution of nonlocality ellipses, here we see there are several systematic, largescale patterns in the distributions of mixing ellipse characteristics that align with the system's dynamical regime boundaries. For example, the mixing ellipses tend to be zonally elongated within the jet flow for $10<$ $x<20$ and $40<x<55$, indicating a large along-jet mixing rate and small cross-jet mixing rate. On the jet flanks in the upstream region $(x<28)$, they are increasingly tilted toward the jet, again consistent with the directionality of the eddy covariance ellipses here, which indicate eddy momentum fluxes consistent with barotropic instability. The negative minor axis of ellipses within the jet downstream, indicated by red color, is consistent with the negative meridional diffusivity there. On the outer edge of the recirculation gyres $(10<x<40$ and $5<|y|<10$ ) and inside the jet farther downstream $(x>50)$, the mixing ellipses are relatively large and circular, indicating relatively large and isotropic mixing. Finally, in the wave radiation regime $(10<|y|<15)$, the mixing ellipses have short axes and are very anisotropic, indicating small and highly directional mixing rates.

A direct visualization of the mixing ellipse anisotropy (diagnosed as the ratio between minor and major axes lengths) is shown in Fig. 14a. The ratio ranges from -1.2 to 1.0. Negative ratios correspond to ellipses with positive major axis but negative minor axis, indicating negative diffusivity in the semiminor axis direction. The magnitude of the positive ratios, which varies between zero and one, should be interpreted as quantifying the degree of mixing anisotropy between purely isotropic (ratio
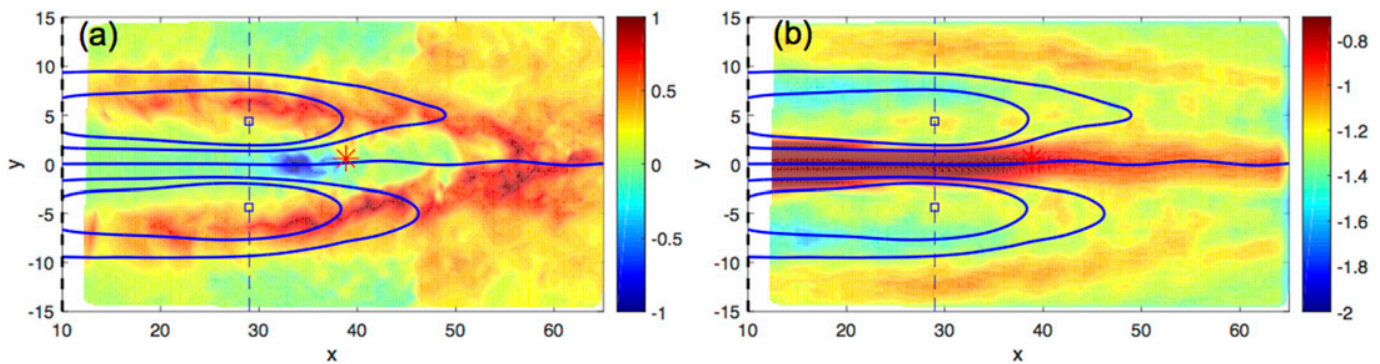

FIG. 14. (a) The ratio between the semiminor and semimajor axes lengths of the mixing ellipses, an indicator of the degree of mixing anisotropy. (b) The magnitude of the spatial gradient of $\bar{q}$ on a logarithmic scale, where $\bar{q}$ is the time-mean PV defined in Eq. (14). The unit of $\bar{q}$ is ndtu ${ }^{-1}$. Annotations denoting key features of the time-mean circulation and EKE distribution are as in Fig. 1. 
magnitude of one) and purely unidirectional (ratio magnitude of zero). Mixing is most anisotropic inside the intense jet $(x<55$; with the exception of just upstream of the EKE maximum where the diffusivity is negative in the semiminor axis direction) and the wave radiation region, particularly to the west of $x=55$. This anisotropy is underpinned by the dominant mean zonal flow of the jet, and the rectilinear linear wave motions (in particular the long westward-propagating waves sourced in the jet's meandering centered at the EKE maximum), respectively. In contrast, mixing tends to be isotropic inside the recirculation gyres and in the far downstream region.

\section{b. Interpretation}

\section{1) REgime 1: Within THE JET FLOW}

The mixing ellipse characteristics are highly variable inside the jet and on its flanks along the jet axis from $x=$ 10 to $x=60$. Here, mixing ellipses evolve from being strongly zonally elongated (indicating enhanced along-jet mixing/suppressed cross-jet mixing) in the upstream unstable jet regime where the zonal jet flow is the strongest $(x \lesssim 20)$ to having negative minor axis (indicating, in this case, negative meridional/cross-jet diffusivity) in the region of jet stabilization $(20 \leqq x \leqq 40)$ to being zonally elongated again downstream $(40 \leqq x \leqq 50)$. Farther downstream, they develop a tilt and become increasingly circular (indicating an increasing contribution of meridional/ cross-jet mixing).

These patterns are largely qualitatively consistent with mixing features inside the KE jet identified using a realistic global eddying model at $0.1^{\circ}$ resolution by Chen et al. (2014b). In this realistic KE jet model, cross-jet mixing within the intense KE jet was found to be small relative to the along-jet mixing rate, consistent with the zonally elongated mixing ellipses seen here at $x<20$. Further, negative cross-jet diffusivities/upgradient eddy tracer fluxes were observed, consistent with the negative minor axis lengths of mixing ellipses diagnosed at $20<$ $x<40$. Finally, relatively large cross-jet eddy diffusivities in the downstream region (large relative to their upstream magnitudes) were noted, consistent with the relatively isotropic mixing ellipses seen here in the downstream jet region $(x>50)$.

The highly zonally elongated mixing ellipses/small cross-jet mixing rates within the jet west of $x=20$ can be interpreted using the critical layer theory originating from Green (1970). This theory suggests that, if the mean flow magnitude differs significantly from that of the eddy phase speed in the direction of the mean flow, cross-jet mixing can be suppressed (e.g., Green 1970; Killworth 1997; Ferrari and Nikurashin 2010; Klocker et al. 2012a; Klocker and Abernathey 2014). Chen et al. (2014b) explains the suppression of mixing within the upstream region of the realistic model KE jet using this idea and found that it was relevant to mixing in the intense $\mathrm{KE}$ jet in the region away from topography. Here, we estimate the along-jet phase speed using the Radon approach [see Chen et al. (2014b) for a description] and also find that within the jet west of $x=20$, the phase speed greatly differs from the mean flow magnitude. Specifically, in this area $(x<20$ and $|y|<5)$, the averaged eddy phase speed along the mean jet direction is only $-0.05 \mathrm{ndlu} / \mathrm{ndtu}$ (with the negative value indicating that eddies propagate upstream relative to the mean flow), a small fraction of the averaged mean flow magnitude here equal to $0.34 \mathrm{ndlu} / \mathrm{ndtu}$. Thus, cross-jet mixing suppression arising from strongly mismatched mean flow and eddy phase speed magnitudes appears a reasonable explanation for the mixing suppression observed. Note that besides the mismatch between mean flow and eddy phase speed, the strong eddy anisotropy within the jet west of $x=20$ could also contribute to the mixing anisotropy here. The ratio between $u^{\prime 2}$ and $v^{\prime 2}$ averaged over the area $x<20$ and $|y|<5$ is as large as 4.9 because of the strong meridional gradient of the background potential vorticity there.

Negative values of particle-based, cross-jet eddy diffusivities have been identified in both a realistic western boundary current jet analyzed by Chen et al. (2014b) and the idealized jet in this study. As pointed out by Chen et al. (2014b), Lagrangian and Eulerian eddy diffusivities are consistent if the Lagrangian diffusivity converges (e.g., Davis 1987, 1991; Griesel et al. 2010). Therefore, since Eulerian eddy diffusivities have been found to be negative in the area with upgradient eddy tracer fluxes, it is unsurprising that Lagrangian eddy diffusivities are negative. Chen et al. (2014b) provide an interpretation of negative Lagrangian eddy diffusivity from the particle perspective. In brief, the integrand of Eq. (4), the autocorrelation, can have both positive and negative lobes. If negative lobes cover a larger area than positive ones, Lagrangian eddy diffusivity can be negative. The suppression of cross-jet mixing, that is, the mismatch between mean flow magnitude and eddy phase speed along the mean flow direction contributes to the occurrence of negative lobe. Section 6 from Chen et al. (2014b) provides more details.

The evolving structure of mixing ellipses within the jet flow is also consistent with the along-jet evolution of the jet's dynamics, as discussed in Waterman and Jayne (2011) and Waterman and Hoskins (2013) in a number of significant ways. For example, in the upstream region, the unstable jet is narrow and sharp, imposing a strong PV barrier to cross-jet mixing (Dritschel and McIntyre 2008). Second, the occurrence of negative ellipse 

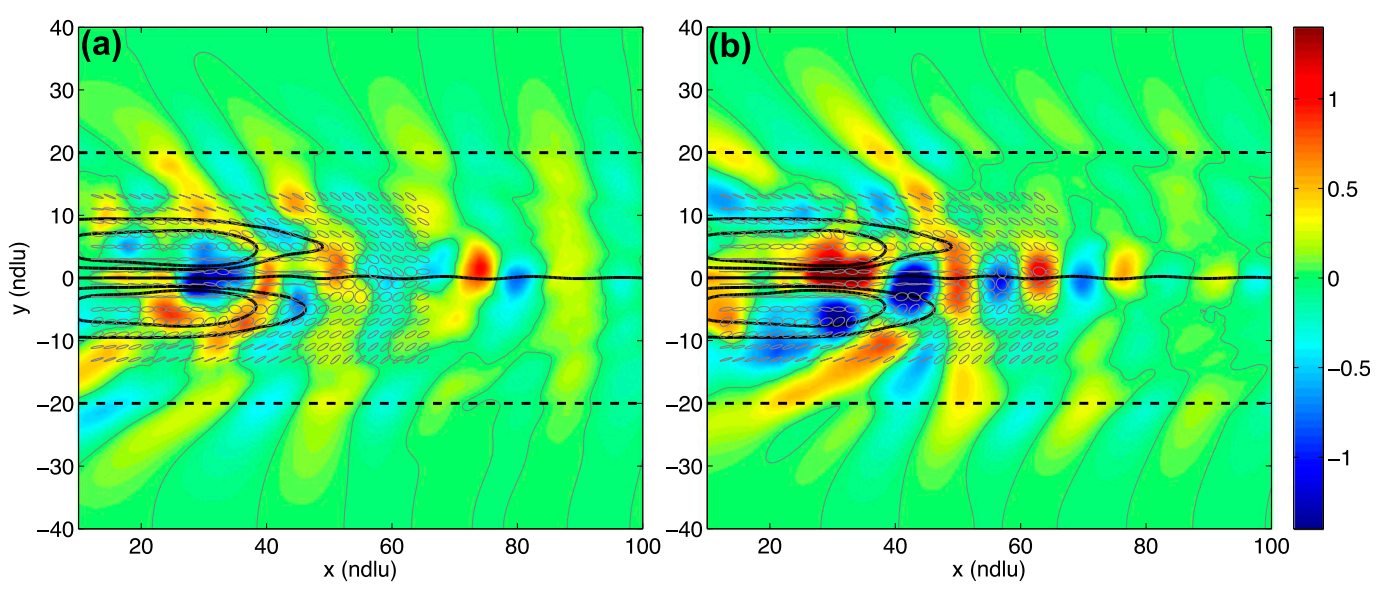

FIG. 15. Representative snapshots of the streamfunction anomalies, that is, the deviation of the streamfunction $\psi$ from its time mean over 5000 ndtus $\left(\mathrm{ndlu}^{2} \mathrm{ndtu}^{-1}\right)$. Solid black lines indicate the time-mean streamfunction averaged over 5000 ndtus. Gray contours are those of the zero values of the $\psi$ anomalies. The horizontal dashed black lines indicate the extent of the northern and southern sponge layers. Gray ellipses contain information about the eccentricity and tilt of the mixing ellipses from Fig. 14. Here, the lengths of major and minor axes are normalized by the length of the major axis, which make relatively small mixing ellipses more visible.

semiminor axis lengths from $20 \leqq x \leqq 40$ is consistent with upgradient eddy momentum fluxes on the jet flanks in the upstream jet region $(12 \leqslant x \leqslant 25)$ and in the downstream wave radiator region $(x \geqslant 28)$. We note that the exact collocation of regions of negative diffusivity diagnosed here and regions of upgradient eddy fluxes are not necessarily expected given the significant nonlocality of mixing inside the jet, which may obscure the distinct transitions between mixing regimes as diagnosed by the mixing ellipse metric.

\section{2) Regime 2: WAVE RADIATION REgION}

As noted above, previous studies of the WBC model (e.g., Waterman and Jayne 2011; Waterman and Hoskins 2013) have argued that the strong meandering of the stabilized jet downstream acts effectively as a localized wave maker in the vicinity of the EKE maximum, radiating Rossby waves that propagate away from the jet. In the model run considered here, we can clearly see these waves in instantaneous snapshots of the streamfunction field, where they appear as the dominant flow field feature outside of the jet and time-mean recirculation gyres (Fig. 15). In this region, the mixing ellipses are relatively small, anisotropic, and roughly align with the wave streamfunction (Figs. 13, 14, 15). This is consistent with an eddy field model consisting of a single propagating wave, in which eddy motions align with the wave streamfunction contours, and thus mixing only occurs in the direction perpendicular to the wavenumber vector. This alignment is also predicted by an application of the multiwavenumber mixing theory from Chen et al. (2015b) to the idealized single wave scenario (see appendix B). Note that our results here also indicate that a single propagating wave can also mix properties in the direction aligned with the wave streamfunction.

\section{3) Regime 3: ReCIRCULATION GYRE REGION}

Inside the time-mean recirculations, the time-mean $\mathrm{PV}$ gradient is small, and $\mathrm{PV}$ is quasi homogenized (Fig. 14b). The mechanism of the time-mean PV homogenization is introduced by Rhines and Young (1982). The relatively isotropic mixing ellipses in this region, shown in Figs. 13 and 14a, are consistent with the small time-mean PV gradient here (Fig. 14b). The detailed rationale is as follows: The total flow can be decomposed into a time-mean part, denoted by ', and an anomaly component, denoted by ${ }^{\prime}$. Following previous studies (e.g., Wang et al. 2013), assuming the time-mean basic state is forced by the steady inflow at the western boundary, we can rewrite the model's governing equation [Eq. (2)] as

$\frac{\partial}{\partial t} \nabla^{2} \psi^{\prime}+J\left(\bar{\psi}+\psi^{\prime}, \nabla^{2} \psi^{\prime}\right)+J\left(\psi^{\prime}, \bar{q}\right)=-R \nabla^{2} \psi^{\prime}$.

Here, $\bar{\psi}$ is the time-mean streamfunction, and $\bar{q}$ is the time-mean PV:

$$
\bar{q}=\beta y+\frac{\partial}{\partial x} \bar{v}-\frac{\partial}{\partial y} \bar{u}
$$

where $\bar{u}$ and $\bar{v}$ are time-mean zonal and meridional velocities. In the linear limit, the eddy phase speed along the mean flow direction is 


$$
C_{\text {eddy }}=\sqrt{\bar{u}^{2}+\bar{v}^{2}}-\frac{\bar{q}_{n} k}{k^{2}+l^{2}},
$$

where $\bar{q}_{n}$ is the time-mean PV gradient in the acrossmean flow direction, and $k$ and $l$ are wavenumbers in the along- and across-mean flow directions, respectively. In the recirculation gyre region, $\bar{q}$ is quasi homogenized and thus $\bar{q}_{n}$ is approximately zero. Therefore, eddies do not propagate relative to the mean flow. That is, they are simply advected by the mean flow. In this scenario, critical layer theory indicates that mixing across the mean flow direction is not suppressed (e.g., Ferrari and Nikurashin 2010), and mixing is quasi isotropic.

If we assume that the spatial scale of the $\bar{u}$ and $\bar{v}$ variation is large, then in the recirculation gyre region, Eq. (13), with negligible $J\left(\psi^{\prime}, \bar{q}\right)$ caused by PV homogenization, is Galilean invariant (Chen and Flierl 2015). That is, in the moving coordinate ( $\left.x^{\prime}=x-\bar{u} t, y^{\prime}=y-\bar{v} t\right)$, Eq. (13) is reduced to the two-dimensional turbulence equation with neither $\beta$ effect nor mean flow (Chen and Flierl 2015), and eddies in this system are isotropic (Rhines 1979). Note that this work provides a discussion about particle-based mixing anisotropy in a recirculation gyre, which involves closed streamlines. For discussions about how passive scalars (e.g., potential vorticity) mix within closed streamlines, readers can refer to Rhines and Young (1983).

\section{Summary and discussion}

Using a barotopic, QG model with a jet flow injected from the western boundary, we have examined various properties of eddy mixing in this zonally evolving jet system using analyses derived from the dispersion of numerical particles, specifically the nonlocality of mixing and mixing anisotropy. The parameters of the model setup are based on a typical WBC jet, and eddy-mean flow processes in this model are relevant to realistic WBCs (e.g., Waterman and Jayne 2011; Waterman et al. 2011). This work complements our previous fundamental understanding of the role of eddies in barotropic jet systems (e.g., Waterman and Jayne 2011) by considering Lagrangian and mixing perspectives.

To assess the nonlocality of mixing, we estimated nonlocality ellipses, which depict the spreading distance of particles for converged diffusivity estimates. We found that the size, shape, and tilt of these ellipses have strong spatial variability. However, at a given location, the four nonlocality ellipses corresponding to the four components of $\kappa_{i j}$ are similar. For a jet dimensionalized to mimic the Kuroshio Extension, the nonlocality ellipses are characterized by dimensional semimajor and semiminor axis lengths ranging from 0 to 240 and from 0 to $160 \mathrm{~km}$, respectively. The dimensional equilibration time of the diffusivity tensor was found to range from 5 to 40 days. Despite the simplicity of this barotropic, QG model, these numbers are on the same order of magnitude as those diagnosed in the Kuroshio Extension region from a realistic global eddying model (Chen et al. 2014b). Our simple model indicates the degree of mixing nonlocality in this system scales with the equilibration time and eddy velocity [Eq. (12)].

The characteristics of mixing ellipses depend on the flow regime. In the wave radiation region, mixing ellipses are elongated and align with the wave streamfunction. These ellipse characteristics can be explained by a simple mixing theory based on the linear and homogeneous assumption (appendix B). In the recirculation gyre region, the time-mean PV is roughly homogenized, leading to quasi-isotropic turbulence and mixing ellipses. Mixing ellipses within the jet flow have strong zonal variability. In the upstream region of the jet, ellipses are zonally elongated because of the suppression of cross-jet mixing by the intense jet and the anisotropy of eddy velocity. Inside the jet downstream, eddy fluxes are upgradient (e.g., Waterman and Jayne 2011), and consistently crossjet eddy diffusivities are negative. Thus, the minor axis of ellipses has negative length. Inside the jet farther downstream, cross-jet eddy diffusivities switch from being negative to being positive, and ellipses are zonally elongated because of the small values of cross-jet eddy diffusivities as they change from negative to positive. Similar to nonlocality ellipses, eddy diffusivities in this idealized model also have similarities to those in a realistic model of the Kuroshio Extension (Chen et al. 2014b), such as negative cross-jet eddy diffusivities in the area with upgradient eddy PV fluxes and the smaller ratio between cross-jet and along-jet eddy diffusivities in the intense jet area.

This work illustrates how to estimate nonlocality ellipses and mixing ellipses in a jet system. We extended the clustering adaptive bin approach (e.g., Chen et al. 2014b) by taking into account the fast advection of the jet and mixing nonlocality. The method we use here differs from that in Chen et al. (2014b) in the following aspects: 1) estimating as local as possible diffusivities by choosing the time integration limits in the diffusivity calculation to be a function of the equilibration time of $\left.\kappa_{i j}, 2\right)$ using forward pseudotrajectories only, 3) remapping the diffusivity estimate from the bin centroid to the track centroid, and 4) extending diffusivity estimates from its along-/cross-jet components to the full diffusivity tensor. This work also reveals that, to thoroughly quantify mixing, one must characterize both the diffusivity tensor and the mixing nonlocality.

This work can be extended in several avenues. First, it would be useful to evaluate the sensitivity of mixing 
properties to the choice of model parameters, such as the meridional gradient of planetary vorticity and bottom friction. Second, the methodology to estimate nonlocality and mixing ellipses presented in this work can also be applied to other geophysical scenarios (e.g., realistic global eddying models and satellite observations of both the atmosphere and the ocean). For example, the model employed in this study, though proven to be very useful, does not take into account baroclinicity or the effect of eddies and waves propagating into the WBC from other parts of the ocean. A more realistic model, for example, the multiple-layer double gyre model that Kamenkovich et al. (2015) chose to study mixing, would help us to evaluate the effect of these factors on mixing properties. An understanding of the degree of mixing nonlocality obtained from these efforts would be useful for further improvement of eddy parameterization schemes. Third, numerical particles are analogous to passive tracers, which have negligible diffusion and sources/sinks. However, key climate tracers (e.g., carbon and heat) are not passive. The dependence of mixing nonlocality on the tracer type is still to be revealed. Fourth, the dependence of mixing anisotropy on flow regime is still to be assessed in realistic ocean scenarios. Finally, the diagnosis of nonlocality ellipses, computed here for single-particle diffusivity estimates, can be extended to the case of relative dispersion.

Results in this study have implications for eddy parameterizations in the ocean components of standard climate models, whose resolutions are too coarse to resolve all the eddies. First, most eddy parameterizations are based on the local assumption, that is, they link eddy tracer fluxes with the local large-scale tracer gradient only. However, if the model resolution is finer than the length of the major/minor axis of the nonlocality ellipse, the local assumption inherent in standard eddy parameterization schemes is not valid. Yet, realistically including the nonlocality effect in eddy parameterizations is challenging and more work needs to be done: 1) the degree of nonlocality varies with both space and the component of $\kappa_{i j}^{\infty}$ even in this idealized model and 2) the relation between the nonlocality ellipses and the local Eulerian diffusivities used in coarse-resolution models can be complicated. Second, consistent with Wilson and Williams (2006), in both realistic simulations (Chen et al. 2014b) and this idealized barotropic QG model, negative diffusivities are prevalent in the regions of eddy energy decay (e.g., western boundary current extensions). The prevalence of negative diffusivities indicates that we should consider their effect in coarse-resolution climate models. Third, strong anisotropy of mixing inside the jet and in the wave radiation region suggests there is a need to implement an anisotropic mixing coefficient in coarseresolution models.

Acknowledgments. The authors thank Steven Jayne for the continued use of his WBC jet model and for all of his insight into its workings over the years. S. Waterman gratefully acknowledges support from the National Science and Engineering Research Council of Canada (Grant 22R23085), the Alfred P. Sloan Foundation, and the University of British Columbia.

\section{APPENDIX A}

\section{Eccentricity and Tilt for Momentum Ellipses}

For the convenience of the reader, here we briefly illustrate how to estimate the eccentricity and tilt of momentum ellipses from momentum fluxes.

\section{a. Eddy momentum ellipses}

Waterman and Lilly (2015) discuss the eccentricity and tilt of the variance ellipse, termed here the eddy momentum ellipse. In brief, the derivations of ellipse properties start from the horizontal velocity covariance matrix:

$$
\mathbf{M}_{e}=\left[\begin{array}{ll}
\overline{u^{\prime 2}} & \overline{u^{\prime} v^{\prime}} \\
\overline{u^{\prime} v^{\prime}} & \overline{v^{\prime 2}}
\end{array}\right],
$$

where - denotes the temporal average, and $\cdot^{\prime}$ denotes the deviation from the time mean. As discussed in Waterman and Lilly (2015), $\mathbf{M}_{e}$ defines an ellipse. Here, we define its semimajor axis length as $a_{e}$, its semiminor axis length as $b_{e}$, and the angle of the major axis measured anticlockwise from the positive $x$ direction as $\theta_{e}$. The terms $a_{e}, b_{e}$, and $\theta_{e}$ can be obtained following the procedure from Preisendorfer (1988) and Morrow et al. (1994). The eccentricity of this ellipse $E_{e}$ measures ellipse anisotropy and is given by

$$
E_{e}=\frac{a_{e}^{2}-b_{e}^{2}}{a_{e}^{2}+b_{e}^{2}}=\frac{\sqrt{\left(\overline{u^{\prime 2}}-\overline{v^{\prime 2}}\right)^{2}+4\left(\overline{u^{\prime} v^{\prime}}\right)^{2}}}{\overline{u^{\prime 2}}+\overline{v^{\prime 2}}} .
$$

The ellipse tilt $\theta_{e}$ can be obtained from

$$
\tan \left(\theta_{e}\right)=\frac{\sigma_{1, e}^{2}-\overline{u^{\prime} u^{\prime}}}{\overline{u^{\prime} v^{\prime}}},
$$

where

$$
\sigma_{1, e}^{2}=\frac{1}{2}\left[\overline{u^{\prime 2}}+\overline{v^{\prime 2}}+\sqrt{\left(\overline{u^{\prime 2}}-\overline{v^{\prime 2}}\right)^{2}+4\left(\overline{u^{\prime} v^{\prime}}\right)^{2}}\right] .
$$




\section{b. Total momentum ellipses}

For the total momentum ellipse, one derives the ellipse properties from the matrix

$$
\mathbf{M}_{t}=\left[\begin{array}{ll}
\overline{u^{2}} & \overline{u v} \\
\overline{u v} & \overline{v^{2}}
\end{array}\right]
$$

Again, following Preisendorfer (1988), we can obtain an ellipse semimajor axis length $a_{t}$, semiminor axis length $b_{t}$, and tilt $\theta_{t}$, which is the angle of the major axis measured anticlockwise from the positive $x$ direction. The tilt $\theta_{t}$ can be obtained from

$$
\tan \left(\theta_{t}\right)=\frac{\sigma_{1, t}^{2}-\overline{u u}}{\overline{u v}}
$$

where

$$
\sigma_{1, t}^{2}=\frac{1}{2}\left[\overline{u^{2}}+\overline{v^{2}}+\sqrt{\left(\overline{u^{2}}-\overline{v^{2}}\right)^{2}+4(\overline{u v})^{2}}\right] .
$$

The eccentricity $E_{t}$ measures the anisotropy of the total momentum ellipse, and it is given by

$$
E_{e}=\frac{a_{t}^{2}-b_{t}^{2}}{a_{t}^{2}+b_{t}^{2}}=\frac{\sqrt{\left(\overline{u^{2}}-\overline{v^{2}}\right)^{2}+4(\overline{u v})^{2}}}{\overline{u^{2}}+\overline{v^{2}}} .
$$

\section{APPENDIX B}

\section{A Mixing Ellipse Theory for the Wave Radiation Regime}

In the linear and homogeneous system, if the eddy field can be approximated as a single propagating wave (as is the case in the wave radiation regime), the minor axis length of the mixing ellipse approaches zero, and the major axis aligns with the eddy streamfunction contours and thus is perpendicular to the wavenumber vector. Derivation details follow.

Chen et al. (2015b) shows that, if the mean flow is spatially and temporally constant and the eddy velocity magnitude is much smaller than the mean flow magnitude, the eddy diffusivity tensor $\kappa_{i j}^{\infty}$ is linked with the frequency-wavenumber spectra of the eddy velocity:

$$
\kappa_{i j}^{\infty, \text { theory }}(\mathbf{x})=\frac{1}{2} \int_{-\infty}^{\infty} \int_{-\infty}^{\infty} S_{u_{i}^{\prime}, u_{j}^{\prime}}\left(k^{\prime}, l^{\prime}, \bar{u} k^{\prime}+\bar{v} l^{\prime}, \mathbf{x}\right) d k^{\prime} d l^{\prime},
$$

where $S_{u_{i}^{\prime} u_{i}^{\prime}}\left(k^{\prime}, l^{\prime}, \omega, \mathbf{x}\right)$ is the frequency-wavenumber cross spectrum for the eddy velocities in a regular geographic patch centered at the location $\mathbf{x}$. The terms $\bar{u}$ and $\bar{v}$ are mean flow velocities in the zonal and meridional direction, $u_{i}^{\prime}$ denotes the eddy velocity components, and $k^{\prime}$ and $l^{\prime}$ are zonal and meridional wavenumbers.

Now consider the case in which the eddy flow has a single wave form:

$$
\psi^{\prime}(x, y, t)=\operatorname{Re}\{a(t) \exp [i(k x+l y)]\},
$$

where $\psi^{\prime}$ is the eddy streamfunction. In this idealized scenario, the spectra for the eddy velocity can be derived from the spectra of $\psi^{\prime}$, that is, $S_{\psi^{\prime}}$ :

$$
\begin{aligned}
S_{u^{\prime} u^{\prime}} & (k, l, \omega, \mathbf{x})=l^{2} S_{\psi^{\prime}}(k, l, \omega, \mathbf{x}), \\
S_{v^{\prime} v^{\prime}}(k, l, \omega, \mathbf{x}) & =k^{2} S_{\psi^{\prime}}(k, l, \omega, \mathbf{x}), \quad \text { and } \\
S_{u^{\prime} v^{\prime}}(k, l, \omega, \mathbf{x}) & =S_{v^{\prime} u^{\prime}}(k, l, \omega, \mathbf{x}) \\
& =-k l S_{\psi^{\prime}}(k, l, \omega, \mathbf{x}) .
\end{aligned}
$$

Substituting $S_{u_{i}^{\prime} u_{j}^{\prime}}$ from Eqs. (B3) and (B4) into Eq. (B1) gives

$$
\kappa_{x x}^{\infty, \text { theory }}(\mathbf{x})=l^{2} \underbrace{\frac{1}{2} \int_{-\infty}^{\infty} \int_{-\infty}^{\infty} S_{\psi^{\prime}}\left(k^{\prime}, l^{\prime}, \bar{u} k^{\prime}+\bar{v} l^{\prime}, \mathbf{x}\right) d k^{\prime} d l^{\prime}}_{\Pi},
$$

and

$$
\begin{aligned}
& \kappa_{y y}^{\infty, \text { theory }}(\mathbf{x})=k^{2} \Pi, \quad \text { and } \\
& \kappa_{x y}^{\infty, \text { theory }}(\mathbf{x})=\kappa_{y x}^{\infty, \text { theory }}(\mathbf{x})=-k l \Pi .
\end{aligned}
$$

Section $3 c$ describes how to infer the mixing ellipse properties from $\kappa_{i j}^{\infty}$. Following section $3 \mathrm{c}$ and using Eqs. (B5) and (B6), we can obtain the length of semimajor axis $\sigma_{1}^{2}$, the length of semiminor axis $\sigma_{2}^{2}$, and the angle of the major axis measured anticlockwise from the positive $x$ direction $\theta$. The results are

$\sigma_{1}^{2}=\left(k^{2}+l^{2}\right) \Pi, \quad \sigma_{2}^{2}=0, \quad$ and $\quad \tan \theta=-\frac{k}{l}$.

Note that $\mathbf{K}=k \mathbf{i}+l \mathbf{j}$ is the wavenumber vector for the wave field. Since $\tan \theta=-k / l$, the major axis of the mixing ellipse is perpendicular to the wavenumber vector and thus aligns with the contour of the eddy streamfunction $\psi^{\prime}$.

\section{REFERENCES}

Abernathey, R., J. Marshall, M. Mazloff, and E. Shuckburgh, 2010: Enhanced isopycnal mixing at steering levels in the Southern Ocean. J. Phys. Oceanogr., 40, 170-184, https://doi.org/ 10.1175/2009JPO4201.1. 
— D. Ferreira, and A. Klocker, 2013: Diagnostics of isopycnal mixing in a circumpolar channel. Ocean Modell., 72, 1-16, https://doi.org/10.1016/j.ocemod.2013.07.004.

Bachman, S., 2012: A diagnostic suite of models for the evaluation of oceanic mesoscale eddy parameterizations. Ph.D. thesis, University of Colorado, $246 \mathrm{pp}$.

_- and B. Fox-Kemper, 2013: Eddy parameterization challenge suite I: Eady spindown. Ocean Modell., 64, 12-28, https://doi.org/ 10.1016/j.ocemod.2012.12.003.

Bates, M., R. Tulloch, J. Marshall, and R. Ferrari, 2014: Rationalizing the spatial distribution of mesoscale eddy diffusivity in terms of mixing length theory. J. Phys. Oceanogr., 44, 1523 1540, https://doi.org/10.1175/JPO-D-13-0130.1.

Berloff, P., I. Kamenkovich, and J. Pedlosky, 2009: A model of multiple zonal jets in the oceans: Dynamical and kinematical analysis. J. Phys. Oceanogr., 39, 2711-2734, https://doi.org/ 10.1175/2009JPO4093.1.

Bower, A. S., H. T. Rossby, and J. L. Lillibridge, 1985: The Gulf Stream-Barrier or blender? J. Phys. Oceanogr., 15, 24-32, https:// doi.org/10.1175/1520-0485(1985)015<0024:TGSOB > 2.0.CO;2.

Chen, R., and G. R. Flierl, 2015: The contribution of striations to the eddy energy budget and mixing: Diagnostic frameworks and results in a quasigeostrophic barotropic system with mean flow. J. Phys. Oceanogr., 45, 2095-2113, https://doi.org/10.1175/JPO-D-14-0199.1.

- _ - and C. Wunsch, 2014a: A description of local and nonlocal eddy-mean flow interaction in a global eddypermitting state estimate. J. Phys. Oceanogr., 44, 2336-2352, https://doi.org/10.1175/JPO-D-14-0009.1.

_ J. L. McClean, S. T. Gille, and A. Griesel, 2014b: Isopycnal eddy diffusivities and critical layers in the Kuroshio Extension from an eddying ocean model. J. Phys. Oceanogr., 44, 21912211, https://doi.org/10.1175/JPO-D-13-0258.1.

_- G. R. Flierl, and C. Wunsch, 2015a: Quantifying and interpreting striations in a subtropical gyre: A spectral perspective. J. Phys. Oceanogr., 45, 387-406, https://doi.org/ 10.1175/JPO-D-14-0038.1.

$\longrightarrow$, S. T. Gille, J. L. McClean, G. R. Flierl, and A. Griesel, 2015b: A multiwavenumber theory for eddy diffusivities and its application to the southeast Pacific (DIMES) region. J. Phys. Oceanogr., 45, 1877-1896, https://doi.org/10.1175/JPO-D-14-0229.1.

_ A. Thompson, and G. R. Flierl, 2016: Time-dependent eddy-mean energy diagrams and their application to the ocean. J. Phys. Oceanogr., 46, 2827-2850, https://doi.org/10.1175/JPO-D-16-0012.1.

_, S. T. Gille, and J. L. McClean, 2017: Isopycnal eddy mixing across the Kuroshio Extension: Stable versus unstable states in an eddying model. J. Geophys. Res. Oceans, 122, 4329-4345, https://doi.org/10.1002/2016JC012164.

Chiswell, S. M., 2013: Lagrangian time scales and eddy diffusivity at $1000 \mathrm{~m}$ compared to the surface in the South Pacific and Indian Oceans. J. Phys. Oceanogr., 43, 2718-2732, https://doi.org/ 10.1175/JPO-D-13-044.1.

Danabasoglu, G., and J. Marshall, 2007: Effects of vertical variations of thickness diffusivity in an ocean general circulation model. Ocean Modell., 18, 122-141, https://doi.org/10.1016/ j.ocemod.2007.03.006

da Silveira, I. C. A., 2013: Dynamics of Brazil current dipoles: Barotropic instabilities and flow-western boundary interactions. Ph.D. thesis, Oceanographic Institute of University of São Paulo, 160 pp.

Davis, R., 1987: Modeling eddy transport of passive tracers. J. Mar. Res., 45, 635-666, https://doi.org/10.1357/002224087788326803.

_ 1991: Observing the general circulation with floats. Deep-Sea Res., 38A, S531-S571, https://doi.org/10.1016/S0198-0149(12)80023-9.
Dritschel, D., and M. McIntyre, 2008: Multiple jets as PV staircases: The Phillips effect and the resilience of eddy-transport barriers. J. Atmos. Sci., 65, 855-874, https://doi.org/10.1175/2007JAS2227.1.

Ferrari, R., and M. Nikurashin, 2010: Suppression of eddy diffusivity across jets in the Southern Ocean. J. Phys. Oceanogr., 40, 1501-1519, https://doi.org/10.1175/2010JPO4278.1.

Flierl, G. R., P. Malanotte-Rizzoli, and N. J. Zabusky, 1987: Nonlinear waves and coherent vortex structures in barotropic beta-plane jets. J. Phys. Oceanogr., 17, 1408-1438, https://doi.org/ 10.1175/1520-0485(1987)017<1408:NWACVS>2.0.CO;2.

Gille, S. T., K. Speer, J. R. Ledwell, and A. C. N. Garabato, 2007: Mixing and stirring in the Southern Ocean. Eos, Trans. Amer. Geophys. Union, 88, 382-383, https://doi.org/10.1029/2007EO390002.

Green, J. S. A., 1970: Transport properties of the large-scale eddies and the general circulation of the atmosphere. Quart. J. Roy. Meteor. Soc., 96, 157-185, https://doi.org/10.1002/qj.49709640802.

Griesel, A., S. T. Gille, J. Sprintall, J. L. McClean, J. H. LaCasce, and M. E. Maltrud, 2010: Isopycnal diffusivities in the Antarctic Circumpolar Current inferred from Lagrangian floats in an eddying model. J. Geophys. Res., 115, C06006, https://doi.org/ 10.1029/2009JC005821.

, J. L. McClean, S. T. Gille, J. Sprintall, and C. Eden, 2014: Eulerian and Lagrangian isopycnal eddy diffusivities in the Southern Ocean of an eddying model. J. Phys. Oceanogr., 44, 644-661, https://doi.org/10.1175/JPO-D-13-039.1.

Howe, P., K. A. Donohue, and D. R. Watts, 2009: Stream-coordinate structure and variability of the Kuroshio Extension. Deep-Sea Res. I, 56, 1093-1116, https://doi.org/10.1016/j.dsr.2009.03.007.

Jayne, S. R., and Coauthors, 2009: The Kuroshio Extension and its recirculation gyres. Deep-Sea Res. I, 56, 2088-2099, https://doi.org/ 10.1016/j.dsr.2009.08.006.

Kamenkovich, I., P. Berloff, and J. Pedlosky, 2009: Role of eddy forcing in the dynamics of multiple zonal jets in a model of the North Atlantic. J. Phys. Oceanogr., 39, 1361-1379, https://doi.org/ 10.1175/2008JPO4096.1.

— I. I. Rypina, and P. Berloff, 2015: Properties and origins of the anisotropic eddy-induced transport in the North Atlantic. J. Phys. Oceanogr., 45, 778-791, https://doi.org/10.1175/JPO-D-14-0164.1.

Katsumata, K., and H. Yoshinari, 2010: Uncertainties in global mapping of Argo drift data at the parking level. J. Oceanogr., 66, 553-569, https://doi.org/10.1007/s10872-010-0046-4.

Killworth, P. D., 1997: On the parameterization of eddy transfer. Part I: Theory. J. Mar. Res., 55, 1171-1197, https://doi.org/ 10.1357/0022240973224102.

, and C. W. Hughes, 2002: The Antarctic Circumpolar Current as a free equivalent-barotropic jet. J. Mar. Res., 60, 19-45, https://doi.org/10.1357/002224002762341230.

Klocker, A., and R. Abernathey, 2014: Global patterns of mesoscale eddy properties and diffusivities. J. Phys. Oceanogr., 44, 1030-1046, https://doi.org/10.1175/JPO-D-13-0159.1.

— R. Ferrari, and J. H. LaCasce, 2012a: Estimating suppression of eddy mixing by mean flows. J. Phys. Oceanogr., 42, 15661576, https://doi.org/10.1175/JPO-D-11-0205.1.

,,,--- and S. Merrifield, 2012b: Reconciling float-based and tracer-based estimates of eddy diffusivities. J. Mar. Res., 70, 569-602, https://doi.org/10.1357/002224012805262743.

Koszalka, I. M., and J. H. LaCasce, 2010: Lagrangian analysis by clustering. Ocean Dyn., 60, 957-972, https://doi.org/10.1007/ s10236-010-0306-2.

Kraichnan, R. H., 1987: Eddy viscosity and diffusivity: Exact formulas and approximations. Complex Syst., 1, 805-820.

LaCasce, J. H., R. Ferrari, J. Marshall, R. Tulloch, D. Balwada, and K. Speer, 2014: Float-derived isopycnal diffusivities in the 
DIMES experiment. J. Phys. Oceanogr., 44, 764-780, https:// doi.org/10.1175/JPO-D-13-0175.1.

Maximenko, N. A., B. Bang, and H. Sasaki, 2005: Observational evidence of alternating zonal jets in the World Ocean. Geophys. Res. Lett., 32, L12607, https://doi.org/10.1029/2005GL022728.

McClean, J. L., P. M. Poulain, J. W. Pelton, and M. E. Maltrud, 2002: Eulerian and Lagrangian statistics from surface drifters and a highresolution POP simulation in the North Atlantic. J. Phys. Oceanogr., 32, 2472-2491, https://doi.org/10.1175/1520-0485-32.9.2472.

Monin, A. S., and A. M. Yaglom, 1971: Statistical Fluid Mechanics: Mechanics of Turbulence. MIT Press, $782 \mathrm{pp}$.

Morrow, R., R. Coleman, J. Church, and D. Chelton, 1994: Surface eddy momentum flux and velocity variances in the Southern Ocean from Geosat altimetry. J. Phys. Oceanogr., 24, 2050-2071, https:// doi.org/10.1175/1520-0485(1994)024<2050:SEMFAV>2.0.CO;2.

Nakamura, N., 1996: Two-dimensional mixing, edge formation, and permeability diagnosed in an area coordinate. J. Atmos. Sci., 53, 1524-1537, https://doi.org/10.1175/1520-0469(1996)053<1524: TDMEFA $>2.0 . \mathrm{CO} ; 2$.

Oh, I. S., V. Zhurbas, and W. Park, 2000: Estimating horizontal diffusivity in the East Sea (Sea of Japan) and the northwest Pacific from satellite-tracked drifter data. J. Geophys. Res., 105, 64836492, https://doi.org/10.1029/2000JC900002.

Plumb, R. A., and J. D. Mahlman, 1987: The zonally averaged transport characteristics of the GFDL general circulation/ transport model. J. Atmos. Sci., 44, 298-327, https://doi.org/ 10.1175/1520-0469(1987)044<0298:TZATCO > 2.0.CO;2.

Preisendorfer, R. W., 1988: Principal Component Analysis in Meteorology and Oceanography.: Elsevier, 425 pp.

Qian, Y.-K., S. Peng, and Y. Li, 2013: Eulerian and Lagrangian statistics in the South China Sea as deduced from surface drifters. J. Phys. Oceanogr., 43, 726-743, https://doi.org/10.1175/JPO-D-12-0170.1.

Qiu, B., and S. Chen, 2010: Eddy-mean flow interaction in the decadally modulating Kuroshio Extension system. Deep-Sea Res. II, 57, 1098-1110, https://doi.org/10.1016/j.dsr2.2008.11.036.

Rhines, P. B., 1979: Geostrophic turbulence. Annu. Rev. Fluid Mech., 11, 401-441, https://doi.org/10.1146/annurev.fl.11.010179.002153.

- and W. R. Young, 1982: Homogenization of potential vorticity in planetary gyres. J. Fluid Mech., 122, 347-367, https:// doi.org/10.1017/S0022112082002250.

— closed streamlines? J. Fluid Mech., 133, 133-145, https://doi.org/ 10.1017/S0022112083001822.

Rypina, I. I., L. J. Pratt, and M. S. Lozier, 2011: Near-surface transport pathways in the North Atlantic Ocean: Looking for throughput from the subtropical to the subpolar gyre. J. Phys. Oceanogr., 41, 911-925, https://doi.org/10.1175/2011JPO4498.1.

_ I. Kamenkovich, P. Berloff, and L. Pratt, 2012: Eddy-induced particle dispersion in the near-surface Atlantic. J. Phys. Oceanogr., 42, 2206-2228, https://doi.org/10.1175/JPO-D-11-0191.1.

Sallée, J. B., K. Speer, and S. R. Rintoul, 2011: Mean-flow and topographic control on surface eddy-mixing in the Southern Ocean. J. Mar. Res., 69, 753-777, https://doi.org/10.1357/ 002224011799849408.

Samelson, R. M., 1992: Fluid exchange across a meandering jet. J. Phys. Oceanogr., 22, 431-444, https://doi.org/10.1175/ 1520-0485(1992)022<0431:FEAAMJ > 2.0.CO;2.

Shuckburgh, E., H. Jones, J. Marshall, and C. Hill, 2009: Understanding the regional variability of eddy diffusivity in the Pacific sector of the Southern Ocean. J. Phys. Oceanogr., 39, 2011-2023, https://doi.org/10.1175/2009JPO4115.1.

Taylor, G. I., 1922: Diffusion by continuous movements. Proc. London Math. Soc., 20, 196-211, https://doi.org/10.1112/plms/s2-20.1.196.
Thompson, A. F., 2008: The atmospheric ocean: Eddies and jets in the Antarctic Circumpolar Current. Philos. Trans. Roy. Soc. London, 366, 4529-4541, https://doi.org/10.1098/rsta.2008.0196.

- 2010: Jet formation and evolution in baroclinic turbulence with simple topography. J. Phys. Oceanogr., 40, 257-278, https://doi.org/10.1175/2009JPO4218.1.

— impact on transport in the Antarctic Circumpolar Current. J. Phys. Oceanogr., 42, 956-972, https://doi.org/10.1175/JPO-D-11-0135.1.

Tracey, K. L., D. R. Watts, K. A. Donohue, and H. Ichikawa, 2012: Propagation of Kuroshio Extension meanders between $143^{\circ} \mathrm{E}$ and $149^{\circ}$ E. J. Phys. Oceanogr., 42, 581-601, https://doi.org/ 10.1175/JPO-D-11-0138.1.

Vallis, G. K., 2006: Atmospheric and Oceanic Fluid Dynamics: Fundamentals and Large-Scale Circulation. Cambridge University Press, 745 pp.

Venaille, A., G. K. Vallis, and K. S. Smith, 2011: Baroclinic turbulence in the ocean: Analysis with primitive equation and quasigeostrophic simulations. J. Phys. Oceanogr., 41, 16051623, https://doi.org/10.1175/JPO-D-10-05021.1.

Wang, J., M. A. Spall, G. R. Flierl, and P. Malanotte-Rizzoli, 2013: Nonlinear radiating instability of a barotropic eastern boundary current. J. Phys. Oceanogr., 43, 1439-1452, https:// doi.org/10.1175/JPO-D-12-0174.1.

Waterman, S., and S. R. Jayne, 2011: Eddy-mean flow interaction in the along-stream development of a western boundary current jet: An idealized model study. J. Phys. Oceanogr., 41, 682-707, https://doi.org/10.1175/2010JPO4477.1.

— , and B. J. Hoskins, 2013: Eddy shape, orientation, propagation, and mean flow feedback in western boundary current jets. $J$. Phys. Oceanogr., 43, 1666-1690, https://doi.org/10.1175/JPO-D-12-0152.1. , and J. M. Lilly, 2015: Geometric decomposition of eddy feedbacks in barotropic systems. J. Phys. Oceanogr., 45, 10091024, https://doi.org/10.1175/JPO-D-14-0177.1.

_ , N. G. Hogg, and S. R. Jayne, 2011: Eddy-mean flow interaction in the Kuroshio Extension region. J. Phys. Oceanogr., 41, 1182-1208, https://doi.org/10.1175/2010JPO4564.1.

Watts, D. R., K. L. Tracey, J. M. Bane, and T. J. Shay, 1995: Gulf Stream path and thermocline structure near $74^{\circ} \mathrm{W}$ and $68^{\circ} \mathrm{W}$. J. Geophys. Res., 100, 18291-18312, https://doi.org/10.1029/95JC01850.

Wilson, C., and R. G. Williams, 2006: When are eddy tracer fluxes directed downgradient? J. Phys. Oceanogr., 36, 189-201, https://doi.org/10.1175/JPO2841.1.

Wolfram, P. J., and T. D. Ringler, 2017: Quantifying residual, eddy, and mean flow effects on mixing in an idealized circumpolar current. J. Phys. Oceanogr., 47, 1897-1920, https://doi.org/ 10.1175/JPO-D-16-0101.1.

,,- M. E. Maltrud, D. W. Jacobsen, and M. R. Petersen, 2015: Diagnosing isopycnal diffusivity in an eddying, idealized midlatitude ocean basin via Lagrangian, in Situ, Global, HighPerformance Particle Tracking (LIGHT). J. Phys. Oceanogr., 45, 2114-2133, https://doi.org/10.1175/JPO-D-14-0260.1.

Young, W., P. Rhines, and C. Garrett, 1982: Shear-flow dispersion, internal waves and horizontal mixing in the ocean. J. Phys. Oceanogr., 12, 515-527, https://doi.org/10.1175/ 1520-0485(1982)012<0515:SFDIWA $>2.0$. CO 2 .

Zhurbas, V., and I. S. Oh, 2003: Lateral diffusivity and Lagrangian scales in the Pacific Ocean as derived from drifter data J. Geophys. Res., 108, 3141, https://doi.org/10.1029/2002JC001596. , and —, 2004: Drifter-derived maps of lateral diffusivity in the Pacific and Atlantic Oceans in relation to surface circulation patterns. J. Geophys. Res., 109, C05015, https://doi.org/ 10.1029/2003JC002241. 